\title{
Organocatalytic Desymmetrisation of Fittig's Lactones: Deuterium as a Reporter Tag for Hidden Racemisation
}

\author{
Péter Spránitz \\ Petra Sőregi \\ Bence Béla Botlik \\ Máté Berta \\ Tibor Soós* (D)
}

Institute of Organic Chemistry, Research Centre for Natural Sciences, Magyar tudósok körútja 2A, 1117 Budapest, Hungary

soos.tibor@ttk.mta.hu

Published as part of the 50 Years SYNTHESIS - Golden Anniversary Issue

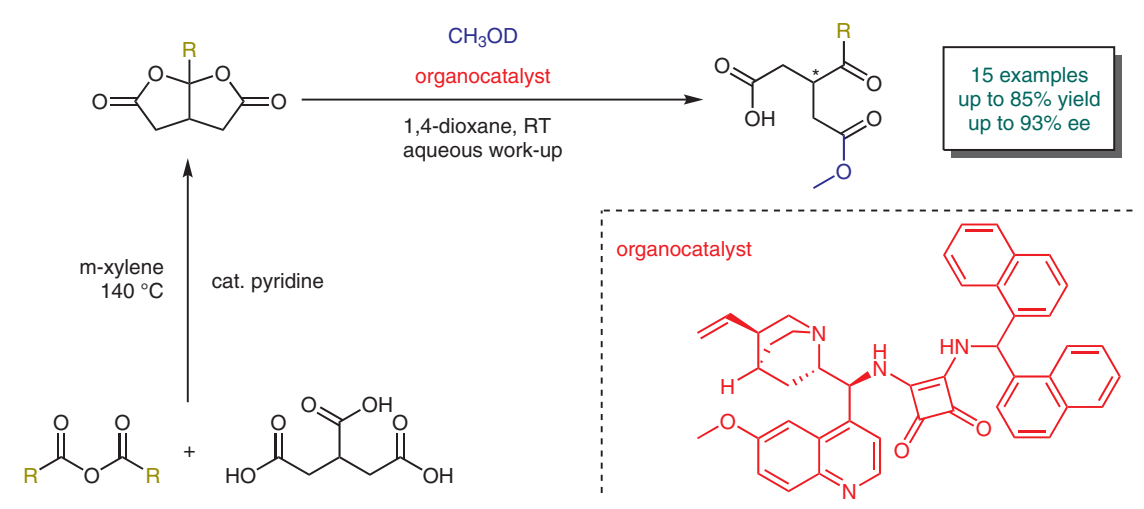

Received: 12.12 .2018

Accepted: 17.12.2018

Published online: 24.01 .2019

DOI: 10.1055/s-0037-1611655; Art ID: ss-2018-z0845-op

License terms: cc)

Abstract Highly enantioselective desymmetrisation of Fittig's lactones with alcohols is promoted by bifunctional cinchona squaramides. The reactions were carried out with monodeuterated methanol to detect possible hidden racemisation of the stereogenic centre. Current evidence suggests that racemisation was not a relevant process for most substrates; partial erosion of enantioselectivity was only detected with ortho-substituted aryl derivates. The resultant glutaric acid derivatives possess a scaffold that is common in natural products and the compounds are also useful chiral building blocks for further synthetic endeavours.

Key words Fittig's lactone, desymmetrization, bislactone acylal, bifunctional organocatalysis, racemization

Over the last decade, the field of enantioselective desymmetrisation mediated by organocatalysts has undergone widespread growth and significantly expanded access to valuable complex chiral molecules. ${ }^{1}$ The influence of this synthetic platform reaches far beyond the domain of asymmetric methodology development; it has a profound impact on total synthesis because of its unique capacity to deliver multifunctional scaffolds tailored to meet the needs of synthetic brevity. ${ }^{2}$ Major efforts in this field have been devoted toward the use of meso or achiral cyclic anhydrides as inexpensive and easily accessible feedstocks (Scheme 1). ${ }^{3}$ Despite the widespread success of these transformations, the substrate scope is limited to five- or six-membered cyclic anhydrides, a feature that can be attributed to both the aforementioned availability and their enhanced reactivity. As a notable exception, Zhu has recently disclosed that less electrophilic $\delta, \delta$-bislactone-acylals could be applied in analogous organocatalytic desymmetrisation. ${ }^{4}$
In a dual effort to extend the narrow breadth of acylalbased substrates and to investigate the synthetic limitation of the organocatalytic desymmetrisation platform, we became interested in developing the asymmetric organocatalytic desymmetrisation of the venerable Fittig's lactones. We envisioned that these easily available $\gamma, \gamma$-bislactone-acylals could be converted into ring-opened products through organocatalytic activation. Herein, we show that bifunc-

Previous work

a) Desymmetrization of cyclic anhydrides

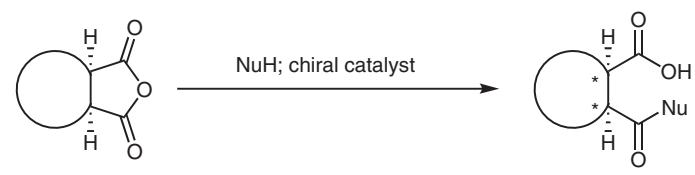

mezo anhydride

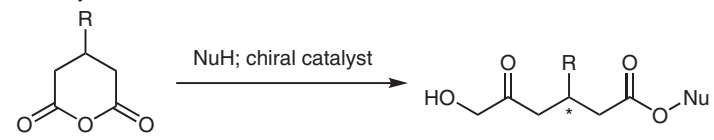

achiral anhydride

b) First bicyclic lactone desymmetrization by Zhu

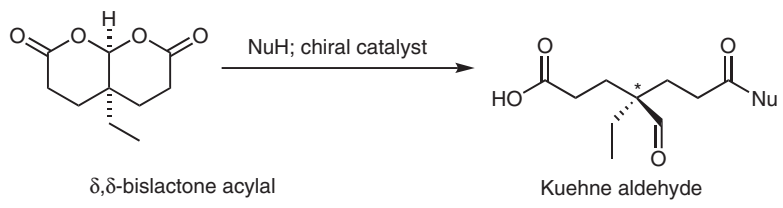

c) This work

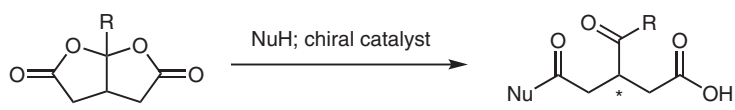

Scheme 1 Organocatalytic strategies for desymmetrisation of acyls 
tional cinchona squaramides can be used to promote the cleavage of this bislactone in an enantioselective manner and to deliver multifunctional chiral building blocks.

The $\gamma, \delta$ - and $\gamma, \gamma$-bislactones are unusual structural elements of many natural products that display important biological activity (Figure 1). ${ }^{5}$ Their bislactone-acylal core shows high stability toward hydrolysis and their structural integrity is not affected by the presence of hydroxyl groups. Furthermore, data concerning the absorption, metabolism, and bioavailability of Bilobalide after oral administration have indicated that the $\gamma, \gamma$-bislactone moiety has a certain stability in biological environments. ${ }^{6}$
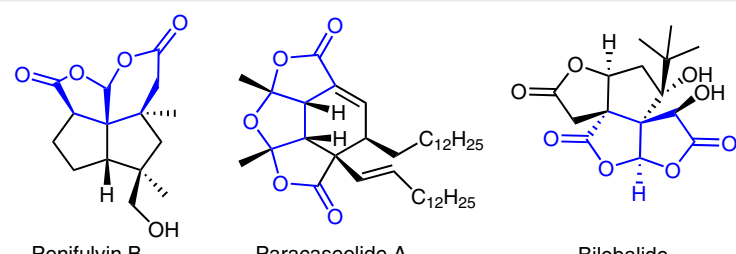

Penifulvin B
Bilobalide

Figure 1 Natural products having lactone-acylal cores

The intrinsic hydrolytic stability of $\gamma, \gamma$-bislactone-acyl$\mathrm{als}^{7}$ of natural products prompted us to study their simplified analogues, the Fittig's lactones, in organocatalytic desymmetrisation. ${ }^{8}$ Reported by Rudolf Fittig in 1901, these lactones can be synthesised from tricarballylic acid in a Dakin-West type reaction. Given that they can be conveniently elaborated, this class of compound has found various uses in polymer chemistry ${ }^{9}$ and total synthesis. ${ }^{10}$ Given all of the above, it is surprising that catalytic desymmetrisation of these lactones as a means to deliver multifunctional chiral building blocks has not previously been exploited, especially in light of their easy accessibility and the current upheaval of desymmetrisation protocols.

Despite the lack of precedent, we postulated that the enantioselective ring opening of Fittig's lactone could be achieved through an organocatalysed, hydrogen-bond-assisted general-base activation strategy. We also recognised that the ability to deliver chiral ring-opened products would hinge on the identification of a catalyst that satisfies two critical requirements: (i) the enantiotopic acyl group discrimination capacity upon ring opening, and (ii) the inability to racemise the acidic $\alpha$-position of the chiral ketone product.

We began our study of the proposed organocatalytic desymmetrisation by examining whether Fittig's lactone was a suitable substrate for desymmetrisation with methanol. Gratifyingly, the bifunctional squaramide organocatalyst 3a enabled the desired transformation of the phenyl-substituted Fittig's lactone 1 at room temperature (Table 1, entry 1 ). To increase the solubility of the applied catalyst, the use of 1 equivalent of $N, N$-diethylacetamide (DEA) was probed as an additive. ${ }^{11}$ We then proceeded to investigate the influence of the applied solvent. This study revealed that etheral

type solvents resulted in higher enantioselectivities and that 1,4-dioxane was the best choice for the desymmetrisation process (entries 1-3). In contrast, neat methanol afforded product 2 with a diminished enantiomeric excess (entry 6), which might be the consequence of the solvent's capacity to disrupt the hydrogen-bond network of the catalyst. A broad range of bifunctional thiourea and squaramide organocatalysts was then evaluated in the model reaction (entries 7-12). The squaramide-type bifunctional organocatalysts ${ }^{12} \mathbf{3 a}-\mathbf{d}$ and $\mathbf{3 f}$ were found to exert higher enantiocontrol than thiourea ${ }^{13}$ catalysts $\mathbf{3 e}$ and $\mathbf{3 g}$. Moreover, installation of a binaphthyl element into the quinine squaramide provided the highest enantioselectivity in the series of benzyl-substituted catalysts (entry 9 vs. entries 3, 7, and 8). Importantly, this is in line with our previous findings on squaramide-promoted organocatalytic Robinson type annulation, ${ }^{14}$ and suggests a direct role of the $\pi$-system in the mechanism of stereoinduction. It is also worth mentioning that quinine-derived catalysts $\mathbf{3} \mathbf{c}$ and $\mathbf{3 e}$ always afforded higher enantioselectivities than their pseudo-enantiomeric quinidine-derived catalysts $\mathbf{3} \mathbf{f}$ and $\mathbf{3 g}$.

A study was then undertaken to elucidate the racemisation capacity of the applied organocatalyst $\mathbf{3 d}$ during the desymmetrisation process, because such a side-reaction might erode the enantioselectivity of the overall process. To identify such a hidden reactivity, we carried out the model reaction with monodeuterated methanol $\left(\mathrm{CH}_{3} \mathrm{OD}\right)$ as a nucleophile and the possible $\mathrm{H}-\mathrm{D}$ exchange was followed by ${ }^{1} \mathrm{H}$ NMR spectroscopy. Most importantly, the same enantioselectivity and no $\mathrm{H}-\mathrm{D}$ exchange was observed in the presence of $\mathrm{CH}_{3} \mathrm{OD}$, which indicates that the applied catalyst does not racemise the stereogenic centre of $\mathbf{2}$. Furthermore, the absence of a dependence of enantioselectivity and yield on the identity of nucleophiles $\left(\mathrm{CH}_{3} \mathrm{OH}\right.$ vs. $\mathrm{CH}_{3} \mathrm{OD}$ ) suggests that the kinetic isotope effect is close to 1 in this organocatalytic process.

We then intended to define the scope of this asymmetric ring-opening methodology; to this end, a variety of Fittig's lactones bearing aromatic, heteroaromatic and also aliphatic substituents were targeted. First, the appropriate Fittig's lactones were synthesised by using the previously reported reaction conditions (Scheme 2). Most of the decarboxylative acylation reactions took place smoothly at high temperature, providing products 4-15 with moderate to high yields. However, the sterically more crowded bislactones $\mathbf{1 6}$ and $\mathbf{1 8}$ formed in this reaction with only lower yields. Furthermore, several attempts under the reported conditions failed to deliver the tert-butyl or 2-thienyl derivatives $\mathbf{1 7}$ and $\mathbf{1 9 .}$

With an optimised desymmetrisation process and the appropriate set of Fittig's lactones in hand, we investigated the scope of the organocatalytic reaction. Notably, we used the relatively inexpensive $\mathrm{CH}_{3} \mathrm{OD}$ as a nucleophile for ring opening to validate and monitor the previously mentioned 
Table 1 Solvent and Catalyst Screen for Desymmetrisation Reaction of $\mathbf{1}^{\text {a }}$
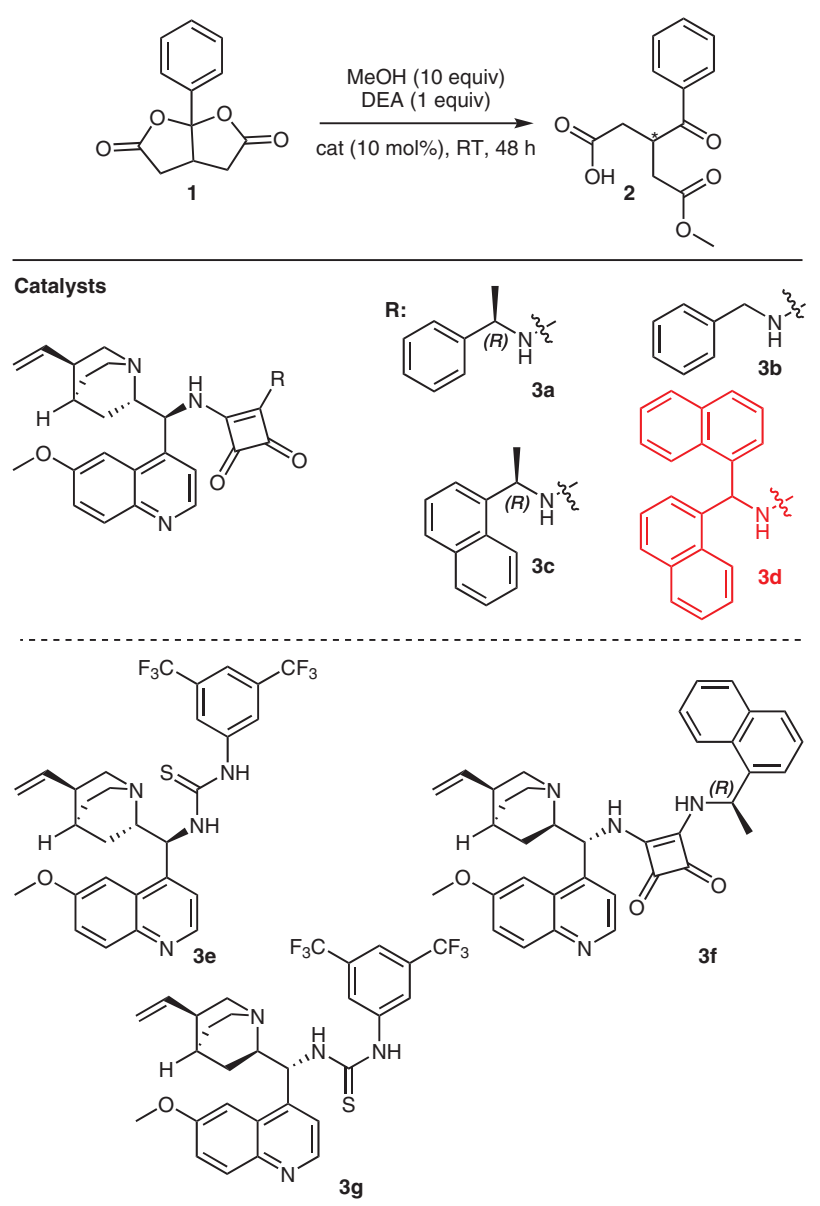

\begin{tabular}{clcc}
\hline Entry & Solvent & Catalyst & ee (\%) \\
\hline 1 & THF & 3a & 76 \\
2 & 2-methyl-THF & 3a & 79 \\
3 & 1,4-dioxane & 3a & 83 \\
4 & toluene & 3a & 50 \\
5 & $\mathrm{CH}_{2} \mathrm{Cl}_{2}$ & $\mathbf{3 a}$ & 56 \\
6 & MeOH & $\mathbf{3 a}$ & 21 \\
7 & 1,4-dioxane & $\mathbf{3 b}$ & 74 \\
8 & 1,4-dioxane & $\mathbf{3 c}$ & 83 \\
9 & 1,4-dioxane & $\mathbf{3 d}$ & 92 \\
10 & 1,4-dioxane & $\mathbf{3 e}$ & 67 \\
11 & 1,4-dioxane & $\mathbf{3 f}$ & -60 \\
12 & 1,4-dioxane & $\mathbf{3 g}$ & -58 \\
\hline
\end{tabular}

${ }^{\mathrm{a}}$ Reactions were performed on $0.5 \mathrm{mmol}$ scale and $0.2 \mathrm{M}$ substrate concentration.

${ }^{\mathrm{b}}$ Determined by chiral HPLC.

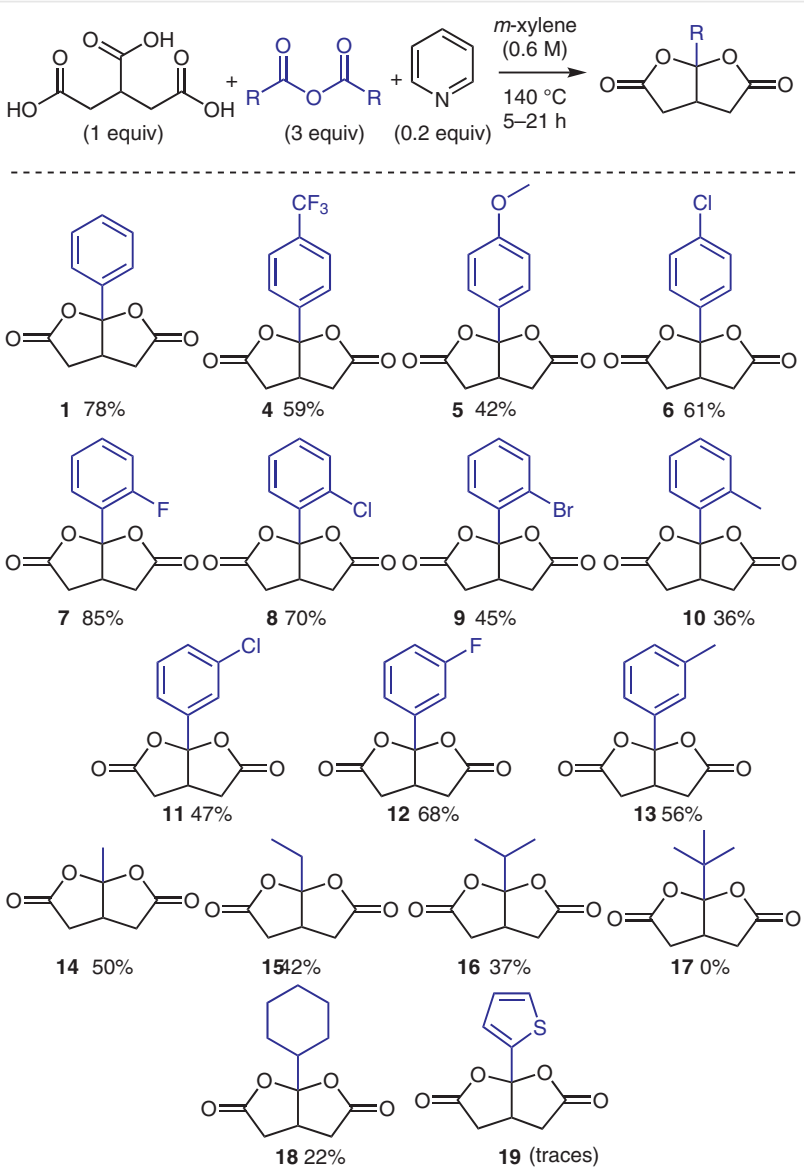

Scheme 2 Substrate scope of Fittig's lactone formation

potential racemisation process of the stereogenic centre upon desymmetrisation (Scheme 3). Aromatic substrates bearing electron-withdrawing or electron-donating groups in the para- or meta-positions underwent smooth ring opening without any detectable H-D exchanges, and afforded the chiral 3-acyl-glutaric acid monomethyl ester derivatives with good yields and high enantioselectivities. In contrast, the desymmetrisation of ortho-substituted aromatic Fittig-lactones 7-10, bearing either electron-withdrawing or electron-donating substituents, resulted in diminished enantioselectivities. The lower ee, however, seems to be the result of the racemisation of the stereogenic centre, because extensive $\mathrm{H}-\mathrm{D}$ exchange was observed by ${ }^{1} \mathrm{H}$ NMR spectroscopy. Variation of aliphatic substituents on the Fittig's lactone backbone revealed that primary and secondary aliphatic substituents were also compatible reaction partners, and afforded the product with good to high enantioselectivities. Finally, we examined the generality of the organocatalytic desymmetrisation with respect to the nucleophilic reaction partner (Scheme 4). Whereas ethanol and benzyl alcohol were highly successful reaction partners, isopropanol and tert-butanol failed to deliver the desired products. 


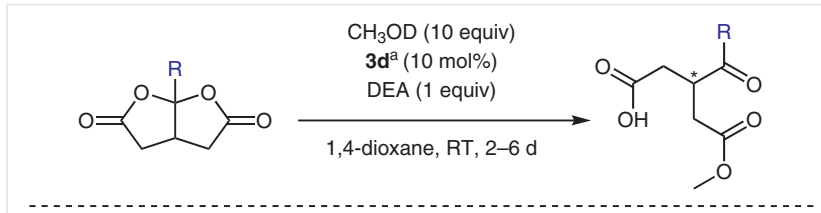<smiles>COC(=O)CC(CC(=O)O)C(=O)c1ccccc1</smiles><smiles>COC(=O)CC(CC(=O)O)C(=O)c1ccc(C(F)(F)F)cc1</smiles><smiles>COC(=O)CC(CC(=O)O)C(=O)c1ccc(Cl)cc1</smiles>

$273 \%$ (92\% ee) $2082 \%(84 \%$ ee $)$ \% ee)<smiles>COC(=O)CC(CC(=O)O)C(=O)c1ccc(OC)cc1</smiles>

e) $2172 \%$ (92\% ee)<smiles>COC(=O)CC(CC(=O)O)C(=O)c1ccccc1Br</smiles>

23 84\% (75\% ee) $24 \quad 77 \%$ (49\% ee) $25 \quad 52 \%$ (37\% ee) $\quad 26 \quad 76 \% \quad$ (74\% ee) $\mathrm{I}=0.82^{\mathrm{b}}$ $\mathrm{I}=0.50^{\mathrm{b}}$<smiles>COC(=O)CC(CC(=O)O)C(=O)c1cccc(Cl)c1</smiles>
$\mathrm{I}=0.23^{\mathrm{b}}$ $\mathrm{I}=0.81^{\mathrm{b}}$<smiles>COC(=O)CC(CC(=O)O)C(=O)c1cccc(C)c1</smiles><smiles>COC(=O)CC(CC(=O)O)C(=O)c1cccc(F)c1</smiles><smiles>COC(=O)CC(=O)C(CC(=O)O)CC(=O)OC</smiles>
(a)<smiles>COC(=O)CC(CC(=O)O)C(=O)C(=O)CC(=O)O</smiles>

$30 \quad 60 \%$ (77\% ee) $\quad 31 \quad 57 \%$ (91\% ee) $\quad 32 \quad 54 \%$ (87\% ee) $\quad 33 \quad 59 \% \quad(92 \%$ ee)

Scheme 3 Substrate scope of desymmetrisation reaction of Fittig's

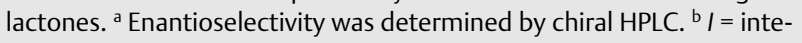
ger of the proton at the stereocentre in ${ }^{1} \mathrm{H}$ NMR spectra.

To better understand the mechanism of this organocatalytic process and the thermodynamic driving force of the transformation, we performed DFT calculations on the reaction of the methyl substituted Fittig's lactone $14 .{ }^{15}$ The calculated free energy of the desymmetrisation process suggests that the ring opening reaction is only slightly exergonic and the actual yield was close to the theoretical yield of the chemical equilibrium of the methanolysis of $\mathbf{1 4}$ (Figure 2). Our theoretical calculations also revealed that $\gamma, \gamma-$ bislactone acylal $\mathbf{1 4}$ is significantly more stable than its glutaric anhydride isomer 40, which explains why this isomer could not be observed experimentally. The generally accepted mechanism of catalysis by cinchona alkaloids in cyclic anhydride desymmetrisation process is general base ac-

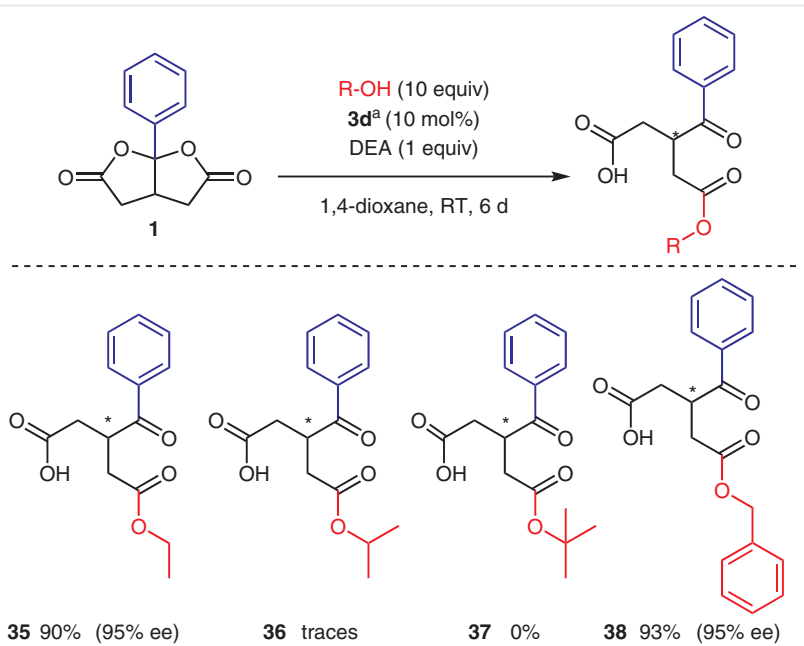

Scheme 4 Desymmetrisation of model Fittig's lactone 1 with different

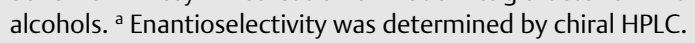

tivation of the methanol by the quinuclidine nitrogen. ${ }^{16}$ Given the size and complexity of the applied catalytic system, we only performed calculations on possible covalent intermediates using a trimethylamine base to gain some insight into the mechanism. For the lactone ring opening, a $\mathrm{B}_{\mathrm{AC}} 2$-like mechanism ${ }^{17}$ was envisioned, which occurs in two steps. In the first step, a methanol molecule attacks the carbonyl carbon in a base-assisted addition reaction that yields tetrahedral intermediates. As calculations revealed, attack of methanol from the convex face is preferred over the concave approach. These intermediates (I2a and $\mathbf{I 2 b}$ ) are metastable and decompose in the second step to a more stable hemiacetal 39.

Taken together, the data presented above indicate that the use of bifunctional squaramides has allowed the development of catalytic desymmetrisation of Fittig's lactones with high enantioselectivity. Thus, the currently narrow scope of bislactone acylal desymmetrisation can be expanded toward substrates having sluggish reactivity. To detect any hidden racemisation process at the stereogenic centre of the product, monodeuterated methanol was used as a nucleophile. We expect this synthetic method to be adopted in total synthesis developments as an efficient platform to generate complex chiral intermediates.

NMR spectra were acquired with a Varian INOVA spectrometer, running at $500 \mathrm{MHz}$ and $125 \mathrm{MHz}$ for ${ }^{1} \mathrm{H}$ and ${ }^{13} \mathrm{C}$, respectively. Chemical shifts $(\delta)$ are reported in ppm relative to residual solvent signals $\left({ }^{1} \mathrm{H}\right.$, $\mathrm{CHCl}_{3}: \delta=7.26$ ppm, DMSO: $\delta=2.50 \mathrm{ppm} ;{ }^{13} \mathrm{C}: \mathrm{CHCl}_{3}: \delta=77.16 \mathrm{ppm}$, DMSO: $\delta=39.52 \mathrm{ppm}$ ). The following abbreviations are used to indicate the multiplicity in spectra: $s$, singlet; $d$, doublet; $t$, triplet; quint., quintet; m, multiplet. ${ }^{13} \mathrm{C}$ NMR spectra were acquired in broad-band continuous decoupled mode. HRMS was performed with a $\mathrm{Q}$ Exactive quadrupol-orbitrap mass spectrometer (Thermo Fischer Scientific, Bremen, Germany). Positive and negative electrospray ionisation 


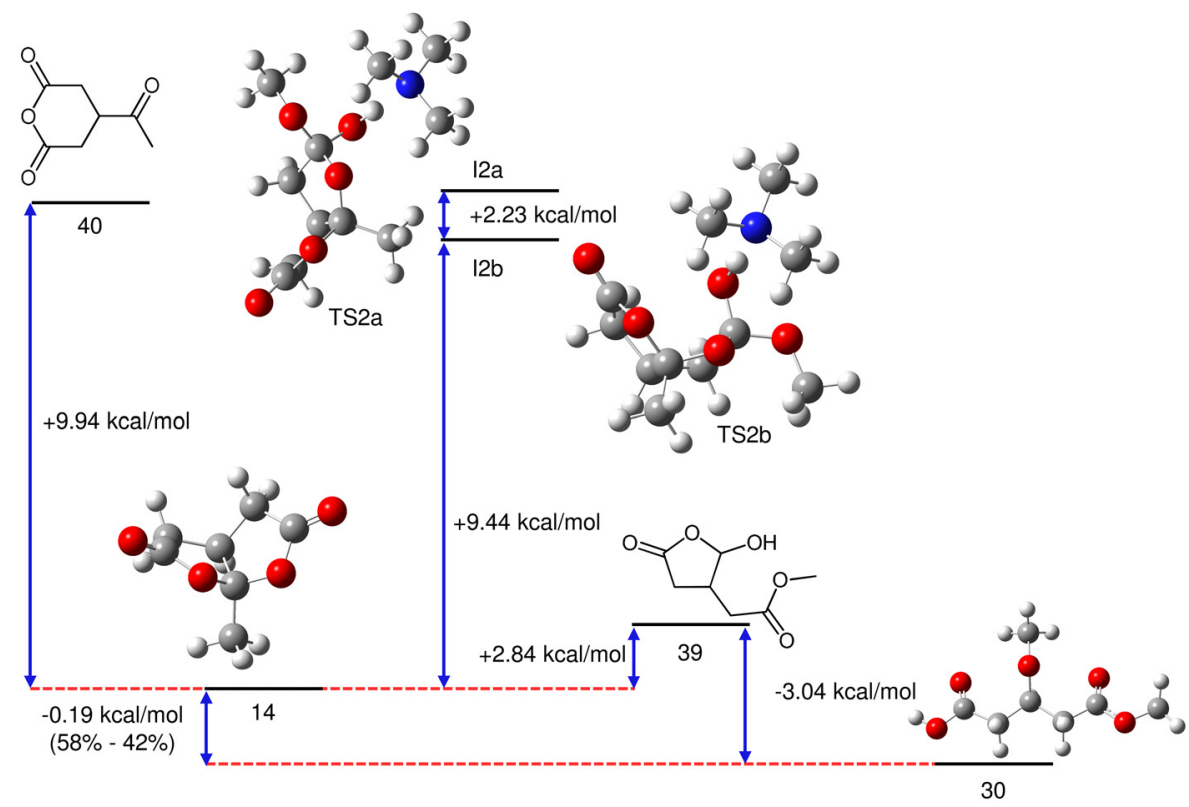

Figure 2 Relevant intermediates of the desymmetrisation of Fittig's lactone $\mathbf{1 4}$ with trimethylamine

mass spectra were acquired by flow injection analysis using $1: 1$ acetonitrile/water solvent mixture containing $0.1 \%$ formic acid $(v / v)$. GCMS analysis was conducted with a Shimadzu GC-2010 Plus Ultra instrument. Melting points were determined with a SRS MPA100 apparatus and are uncorrected. Column chromatography was carried out with a Teledyne ISCO CombiFlash Rf200 UV/VIS system, using RediSep Rf Gold $\circledast$ Normal-phase Silica. The enantiomeric excess of the products were determined with a Jasco ChromPass Chromatography Data System and Waters ${ }^{\mathrm{TM}}$ 600E Multisolvent Delivery System, using chiral stationary phase. Commercially available materials were purchased from Sigma-Aldrich and Fluorochem; all were used without further purification. Ether type solvents and toluene were freshly distilled from sodium/benzophenone.

The conformational analysis involved an initial Monte Carlo sampling using the OPLS_2005 force field as implemented in Schrödinger Maestro. The DFT calculations were carried out with the dispersion corrected, range-separated hybrid $\omega \mathrm{B} 97 \mathrm{X}-\mathrm{D}$ exchange-correlation functional, along with the AUG-cc-pVDZ double- $\zeta$ basis set, as implemented in the Gaussian 16 package. ${ }^{18}$ For each located structure, singlepoint energies were calculated as well with the larger, AUG-cc-pVTZ triple- $\zeta$ basis set. In all DFT calculations, the ultrafine integration grid was employed to warrant the accuracy of numerical integration. The thermal and entropic contributions were estimated within the ideal gas-rigid rotor-harmonic oscillator approximation for $T=298.15 \mathrm{~K}$ and $c=1 \mathrm{~mol} / \mathrm{dm}^{3}$ conditions. The solvent effects were taken into account as well by computing the solvation free energies (at the $\omega \mathrm{B} 97 \mathrm{X}$ D/ AUG-cc-pVDZ level) via the integral equation formalism variant of the polarisable continuum model (IEFPCM).24 calculations were those of the SMD solvation model.

\section{Preparation of Aromatic Anhydrides; General Procedure}

Step 1

In a three-necked $100 \mathrm{~mL}$ flask under $\mathrm{N}_{2}$-atmosphere, the corresponding benzoic acid ( $32 \mathrm{mmol}$ ) was suspended in anhydrous toluene (21 mL, $1.5 \mathrm{M})$. A few drops of anhydrous DMF (126 $\mu \mathrm{L}, 1.6 \mathrm{mmol}$, 0.05 equiv) and $\mathrm{SOCl}_{2}$ ( $3.0 \mathrm{~mL}, 41.5 \mathrm{mmol}, 1.3$ equiv) were added at r.t. sequentially. The mixture was heated to $70{ }^{\circ} \mathrm{C}$ and stirred at this temperature for 1 hour, then cooled to r.t. and the excess $\mathrm{SOCl}_{2}$ was purged from the system by flushing with $\mathrm{N}_{2}$ for $15 \mathrm{~min}$. The solution of the benzoyl chloride was used without purification in the next step.

Step 2

In a three-necked flask under $\mathrm{N}_{2}$-atmosphere, the corresponding benzoic acid ( $32 \mathrm{mmol}, 1.0$ equiv) was suspended in anhydrous toluene (21 mL, 1.5 M). Anhydrous pyridine ( $3.1 \mathrm{~mL}, 38.3 \mathrm{mmol}, 1.2$ equiv) was added to the mixture and the resulting solution was cooled to $0{ }^{\circ} \mathrm{C}$. A solution of the corresponding benzoyl chloride from Step 1 was added at such rate that the temperature was kept under $20^{\circ} \mathrm{C}$, while a white precipitate formed. The resulting suspension was heated at reflux for $2 \mathrm{~h}$ then cooled to r.t. The precipitate $(\mathrm{Pyr} \cdot \mathrm{HCl})$ was filtered, washed with toluene, and the filtrate was evaporated. The residue was dissolved in $\mathrm{CH}_{2} \mathrm{Cl}_{2}(100 \mathrm{~mL})$, washed with $10 \% \mathrm{HCl}(10 \mathrm{~mL})$ then washed with cc. $\mathrm{NaHCO}_{3}(3 \times 10 \mathrm{~mL})$ and brine $(10 \mathrm{~mL})$. The organic layer was dried over $\mathrm{Na}_{2} \mathrm{SO}_{4}$, filtered and evaporated to dryness. Pure anhydrides were obtained in 91-98\% yield; NMR data were consistent with the reported values.

\section{Preparation of Fittig's Lactones; General Procedure}

Into a one-necked $25 \mathrm{~mL}$ flask, tricarballylic acid (518 mg, $2.95 \mathrm{mmol}$, 1.0 equiv) and the appropriate anhydride ( $8.83 \mathrm{mmol}, 3$ equiv) were suspended in $m$-xylene ( $5 \mathrm{~mL}, 0.6 \mathrm{M}$ ). Pyridine was added $(47 \mu \mathrm{L}, 0.59$ mmol, 0.2 equiv) in one portion, then the mixture was heated to $140{ }^{\circ} \mathrm{C}$ and stirred until the completion of the reaction was indicated by GC-MS (5-21 h). After cooling to r.t., $\mathrm{CH}_{2} \mathrm{Cl}_{2}(100 \mathrm{~mL})$ was added 
and the mixture was passed through a short pad of Celite to remove insoluble side-products. The solution was washed with saturated $\mathrm{Na}_{2} \mathrm{CO}_{3}$ solution $(2 \times 10 \mathrm{~mL})$ then with brine $(10 \mathrm{~mL})$. The organic phase was dried over $\mathrm{Na}_{2} \mathrm{SO}_{4}$, filtered, and evaporated to dryness. Purification by flash chromatography (hexanes/EtOAc) afforded the pure Fittig lactone.

\section{6a-Phenyldihydrofuro[2,3-b]furan-2,5(3H,6aH)-dione (1)}

Yield: $502 \mathrm{mg}$ (78\%); off-white solid; mp 136-138 ${ }^{\circ} \mathrm{C}$.

${ }^{1} \mathrm{H} \mathrm{NMR}\left(500 \mathrm{MHz}, \mathrm{CDCl}_{3}\right): \delta=7.48-7.45(\mathrm{~m}, 5 \mathrm{H}), 3.36(\mathrm{tt}, J=9.3$, $4.7 \mathrm{~Hz}, 1 \mathrm{H}), 3.10(\mathrm{dd}, J=18.4,9.3 \mathrm{~Hz}, 2 \mathrm{H}), 2.66(\mathrm{dd}, J=18.4,4.7 \mathrm{~Hz}$, $2 \mathrm{H})$.

${ }^{13} \mathrm{C}$ NMR $\left(125 \mathrm{MHz}, \mathrm{CDCl}_{3}\right): \delta=172.5,136.3,130.2,129.04,124.9$, $112.8,41.4,35.2$.

HRMS (ESI): $m / z[M+\mathrm{H}]^{+}$calcd for $\mathrm{C}_{12} \mathrm{H}_{11} \mathrm{O}_{4}$ : 219.0657; found: 219.0644 .

\section{6a-(4-(Trifluoromethyl)phenyl)dihydrofuro[2,3-b]furan- 2,5(3H,6aH)-dione (4)}

Yield: $498 \mathrm{mg}$ (59\%); off-white solid; decomposition at $128-132{ }^{\circ} \mathrm{C}$. ${ }^{1} \mathrm{H}$ NMR $\left(500 \mathrm{MHz}, \mathrm{CDCl}_{3}\right): \delta=7.73(\mathrm{~d}, J=8.0 \mathrm{~Hz}, 2 \mathrm{H}), 7.59(\mathrm{~d}, J=$ $8.0 \mathrm{~Hz}, 2 \mathrm{H}), 3.38-3.32(\mathrm{~m}, 1 \mathrm{H}), 3.12(\mathrm{dd}, J=18.5,9.5 \mathrm{~Hz}, 2 \mathrm{H}), 2.70$ (dd, $J=18.5,4.2 \mathrm{~Hz}, 2 \mathrm{H}$ ).

${ }^{13} \mathrm{C}$ NMR (125 MHz, $\left.\mathrm{CDCl}_{3}\right): \delta=172.0,140.1,132.5$ (q, $\left.J=33.0 \mathrm{~Hz}\right)$, 126.1 (q, $J=3.64 \mathrm{~Hz}), 123.5$ (q, $J=272.6 \mathrm{~Hz}), 111.8,41.4,35.1$.

HRMS (ESI): $m / z[M+H]^{+}$calcd for $\mathrm{C}_{13} \mathrm{H}_{10} \mathrm{~F}_{3} \mathrm{O}_{4}$ : 287.0531; found: 287.0521

\section{6a-(4-Methoxyphenyl)dihydrofuro[2,3-b]furan-2,5(3H,6aH)-di- one (5)}

Yield: $307 \mathrm{mg}$ (42\%); off-white solid; decomposition at $128-136{ }^{\circ} \mathrm{C}$. ${ }^{1} \mathrm{H}$ NMR $\left(500 \mathrm{MHz}, \mathrm{CDCl}_{3}\right): \delta=7.37(\mathrm{~d}, J=8.9 \mathrm{~Hz}, 2 \mathrm{H}), 6.95(\mathrm{~d}, J=$ $8.8 \mathrm{~Hz}, 2 \mathrm{H}), 3.84(\mathrm{~s}, 3 \mathrm{H}), 3.34(\mathrm{tt}, J=9.3,4.7 \mathrm{~Hz}, 1 \mathrm{H}), 3.08(\mathrm{dd}, J=$ $18.4,9.3 \mathrm{~Hz}, 2 \mathrm{H}$ ), 2.64 (dd, $J=18.4,4.6 \mathrm{~Hz}, 2 \mathrm{H}$ ).

${ }^{13} \mathrm{C}$ NMR $\left(125 \mathrm{MHz}, \mathrm{CDCl}_{3}\right): \delta=172.4,126.4,114.3,55.4,41.4,35.3$. HRMS (ESI): $m / z[M+H]^{+}$calcd for $\mathrm{C}_{13} \mathrm{H}_{13} \mathrm{O}_{5}$ : 249.0763; found: 249.0747.

\section{6a-(4-Chlorophenyl)dihydrofuro[2,3-b]furan-2,5(3H,6aH)-dione} (6)

Yield: 453 (61\%); off-white solid; mp $127-135{ }^{\circ} \mathrm{C}$.

${ }^{1} \mathrm{H}$ NMR (500 MHz, DMSO- $\left.d_{6}\right): \delta=7.60-7.49(\mathrm{~m}, 4 \mathrm{H}), 3.45(\mathrm{~m}, 1 \mathrm{H})$, 3.17 (dd, $J=18.5,9.8 \mathrm{~Hz}, 2 \mathrm{H}), 2.84$ (dd, $J=18.5,3.8 \mathrm{~Hz}, 2 \mathrm{H}$ ).

${ }^{13} \mathrm{C}$ NMR (125 MHz, DMSO): $\delta=174.3,136.7,135.0,129.2,127.7$, $112.4,40.9,35.0$.

HRMS (ESI): $m / z[\mathrm{M}+\mathrm{H}]^{+}$calcd for $\mathrm{C}_{12} \mathrm{H}_{10} \mathrm{ClO}_{4}$ : 253.0268; found: 253.0252 .

\section{6a-(2-Fluorophenyl)dihydrofuro[2,3-b]furan-2,5(3H,6aH)-dione}

(7)

Yield: $592 \mathrm{mg}$ (85\%); off-white solid; decomposition at $100-110^{\circ} \mathrm{C}$.

${ }^{1} \mathrm{H} \mathrm{NMR}\left(500 \mathrm{MHz}, \mathrm{CDCl}_{3}\right): \delta=7.61(\mathrm{td}, J=7.9,1.8 \mathrm{~Hz}, 1 \mathrm{H}$ ), 7.45 (tdd, $J=7.4,5.2,1.8 \mathrm{~Hz}, 1 \mathrm{H}), 7.25-7.05(\mathrm{~m}, 2 \mathrm{H}), 3.54(\mathrm{ttd}, J=10.2,4.4$, $1.5 \mathrm{~Hz}, 1 \mathrm{H}$ ), 3.16 (dd, $J=18.7,10.2 \mathrm{~Hz}, 2 \mathrm{H}$ ), 2.65 (ddd, $J=18.8,4.4$, $1.5 \mathrm{~Hz}, 2 \mathrm{H})$.
${ }^{13} \mathrm{C}$ NMR $\left(125 \mathrm{MHz}, \mathrm{CDCl}_{3}\right): \delta=172.3,159.8(\mathrm{~d}, J=248.3 \mathrm{~Hz}), 132.4(\mathrm{~d}$, $J=9.1 \mathrm{~Hz}), 127.1$ (d, $J=2.1 \mathrm{~Hz}), 124.5$ (d, $J=3.8 \mathrm{~Hz}), 124.3$ (d, $J=$ $10.5 \mathrm{~Hz}), 116.5(\mathrm{~d}, J=21.2 \mathrm{~Hz}), 110.7(\mathrm{~d}, J=1.4 \mathrm{~Hz}), 39.9(\mathrm{~d}, J=2.8 \mathrm{~Hz})$, $35.6(\mathrm{~d}, J=1.9 \mathrm{~Hz})$.

HRMS (ESI): $m / z[M+\mathrm{H}]^{+}$calcd for $\mathrm{C}_{12} \mathrm{H}_{10} \mathrm{FO}_{4}$ : 237.0563; found: 237.5559 .

6a-(2-Chlorophenyl)dihydrofuro[2,3-b]furan-2,5(3H,6aH)-dione

(8)

Yield: $522 \mathrm{mg}$ (70\%); off-white solid; decomposition at $134-142{ }^{\circ} \mathrm{C}$.

${ }^{1} \mathrm{H}$ NMR $\left(500 \mathrm{MHz}, \mathrm{CDCl}_{3}\right): \delta=7.71(\mathrm{dd}, J=7.8,1.8 \mathrm{~Hz}, 1 \mathrm{H}), 7.50(\mathrm{dd}$, $J=7.9,1.4 \mathrm{~Hz}, 1 \mathrm{H}), 7.43(\mathrm{td}, J=7.6,1.7 \mathrm{~Hz}, 1 \mathrm{H}), 7.37(\mathrm{td}, J=7.6$, $1.4 \mathrm{~Hz}, 1 \mathrm{H}), 3.76(\mathrm{tt}, J=10.0,4.3 \mathrm{~Hz}, 1 \mathrm{H}), 3.11(\mathrm{dd}, J=18.6,9.9 \mathrm{~Hz}$, $2 \mathrm{H}), 2.66$ (dd, $J=18.7,4.4 \mathrm{~Hz}, 2 \mathrm{H}$ ).

${ }^{13} \mathrm{C}$ NMR $\left(125 \mathrm{MHz}, \mathrm{CDCl}_{3}\right): \delta=172.4,133.2,132.0,131.7,131.6$, $127.7,127.3,111.8,39.0,35.4$.

HRMS (ESI): $m / z$ [M $+\mathrm{H}]^{+}$calcd for $\mathrm{C}_{12} \mathrm{H}_{10} \mathrm{ClO}_{4}$ : 253.0268; found: 253.0261 .

\section{6a-(2-Bromophenyl)dihydrofuro[2,3-b]furan-2,5(3H,6aH)-dione}

(9)

Yield: $393 \mathrm{mg}$ (45\%); off-white solid; $\mathrm{mp} 162-165^{\circ} \mathrm{C}$.

${ }^{1} \mathrm{H}$ NMR $\left(500 \mathrm{MHz}, \mathrm{CDCl}_{3}\right): \delta=7.72(\mathrm{dd}, J=3.3,1.6 \mathrm{~Hz}, 1 \mathrm{H}), 7.70(\mathrm{dd}$, $J=3.4,1.5 \mathrm{~Hz}, 1 \mathrm{H}), 7.41(\mathrm{td}, J=7.6,1.3 \mathrm{~Hz}, 1 \mathrm{H}), 7.33(\mathrm{td}, J=7.7$, $1.7 \mathrm{~Hz}, 1 \mathrm{H}), 3.80(\mathrm{tt}, J=9.9,4.4 \mathrm{~Hz}, 1 \mathrm{H}), 3.09(\mathrm{dd}, J=18.6,9.9 \mathrm{~Hz}$, $2 \mathrm{H}), 2.65$ (dd, $J=18.6,4.3 \mathrm{~Hz}, 2 \mathrm{H}$ ).

${ }^{13} \mathrm{C}$ NMR $\left(125 \mathrm{MHz}, \mathrm{CDCl}_{3}\right): \delta=172.5,135.1,134.3,131.9,128.1$, $127.8,120.9,112.2,39.1,35.4$.

HRMS (ESI): $m / z[M+\mathrm{H}]^{+}$calcd for $\mathrm{C}_{12} \mathrm{H}_{10} \mathrm{BrO}_{4}$ : 296.9762; found: 296.9755 .

6a-(o-Tolyl)dihydrofuro[2,3-b]furan-2,5(3H,6aH)-dione (10)

Yield: $246 \mathrm{mg}$ (36\%); off-white solid; decomposition at $90-109{ }^{\circ} \mathrm{C}$. ${ }^{1} \mathrm{H}$ NMR $\left(500 \mathrm{MHz}, \mathrm{CDCl}_{3}\right): \delta=7.43(\mathrm{dd}, J=7.8,1.4 \mathrm{~Hz}, 1 \mathrm{H}), 7.35$ (td, $J=7.5,1.4 \mathrm{~Hz}, 1 \mathrm{H}), 7.30-7.19(\mathrm{~m}, 2 \mathrm{H}), 3.55(\mathrm{dq}, J=9.3,4.7 \mathrm{~Hz}, 1 \mathrm{H})$, 3.03 (dd, $J=18.5,9.3 \mathrm{~Hz}, 2 \mathrm{H}), 2.65$ (dd, $J=18.5,4.6 \mathrm{~Hz}, 2 \mathrm{H}), 2.41$ (s, $3 \mathrm{H})$.

${ }^{13} \mathrm{C}$ NMR $\left(125 \mathrm{MHz}, \mathrm{CDCl}_{3}\right): \delta=172.4,136.1,133.1,132.9,130.4$, 126.2, 125.2, 113.6, 39.4, 35.1, 20.8.

HRMS (ESI): $m / z[M+H]^{+}$calcd for $\mathrm{C}_{13} \mathrm{H}_{13} \mathrm{O}_{4}$ : 233.0814; found: 233.0807.

\section{6a-(3-Chlorophenyl)dihydrofuro[2,3-b]furan-2,5(3H,6aH)-dione} (11)

Yield: $349 \mathrm{mg}$ (47\%); off-white solid; decomposition at $125-130{ }^{\circ} \mathrm{C}$.

${ }^{1} \mathrm{H} \mathrm{NMR}\left(500 \mathrm{MHz}, \mathrm{CDCl}_{3}\right): \delta=7.47-7.36(\mathrm{~m}, 3 \mathrm{H}), 7.35-7.31(\mathrm{~m}, 1 \mathrm{H})$, $3.35(\mathrm{tt}, J=9.5,4.6 \mathrm{~Hz}, 1 \mathrm{H}), 3.11(\mathrm{dd}, J=18.6,9.5 \mathrm{~Hz}, 2 \mathrm{H}), 2.67$ (dd, $J=$ $18.6,4.6 \mathrm{~Hz}, 2 \mathrm{H})$.

${ }^{13} \mathrm{C}$ NMR $\left(125 \mathrm{MHz}, \mathrm{CDCl}_{3}\right): \delta=172.1,138.3,135.2,130.4,130.4$, 125.3, 111.9, 123.1, 41.4, 35.1.

HRMS (ESI): $m / z[\mathrm{M}+\mathrm{H}]^{+}$calcd for $\mathrm{C}_{12} \mathrm{H}_{10} \mathrm{ClO}_{4}$ : 253.0268; found: 253.0261.

6a-(3-Fluorophenyl)dihydrofuro[2,3-b]furan-2,5(3H,6aH)-dione (12)

Yield: $473 \mathrm{mg}$ (68\%); off-white solid; decomposition at $151-157^{\circ} \mathrm{C}$. 
${ }^{1} \mathrm{H}$ NMR $\left(500 \mathrm{MHz}, \mathrm{CDCl}_{3}\right): \delta=7.45(\mathrm{td}, J=7.9,5.6 \mathrm{~Hz}, 2 \mathrm{H}), 7.25-7.13$ $(\mathrm{m}, 3 \mathrm{H}), 3.34$ (tt, $J=9.3,4.6 \mathrm{~Hz}, 1 \mathrm{H}), 3.11(\mathrm{dd}, J=18.5,9.5 \mathrm{~Hz}, 2 \mathrm{H})$, 2.67 (dd, $J=18.5,4.6 \mathrm{~Hz}, 2 \mathrm{H}$ ).

${ }^{13} \mathrm{C}$ NMR $\left(125 \mathrm{MHz}, \mathrm{CDCl}_{3}\right): \delta=172.0,162.9(\mathrm{~d}, J=248.6 \mathrm{~Hz}), 138.7(\mathrm{~d}$, $J=7.3 \mathrm{~Hz}), 130.9(\mathrm{~d}, J=8.1 \mathrm{~Hz}), 120.5(\mathrm{~d}, J=3.3 \mathrm{~Hz}), 117.3(\mathrm{~d}, J=$ $21.1 \mathrm{~Hz}), 112.5(\mathrm{~d}, J=24.0 \mathrm{~Hz}), 41.4,35.1$.

HRMS (ESI): $m / z[M+H]^{+}$calcd for $\mathrm{C}_{12} \mathrm{H}_{10} \mathrm{FO}_{4}$ : 237.0563; found: 237.0556 .

\section{6a-(m-Tolyl)dihydrofuro[2,3-b]furan-2,5(3H,6aH)-dione (13)}

Yield: $383 \mathrm{mg}$ (56\%); off-white solid; decomposition at $92-96{ }^{\circ} \mathrm{C}$.

${ }^{1} \mathrm{H}$ NMR $\left(500 \mathrm{MHz}\right.$, DMSO- $\left.d_{6}\right): \delta=7.51-7.15(\mathrm{~m}, 4 \mathrm{H}), 3.44(\mathrm{tt}, J=9.5$, $4.3 \mathrm{~Hz}, 1 \mathrm{H}$ ), 3.17 (dd, $J=18.6,9.8 \mathrm{~Hz}, 2 \mathrm{H}$ ), 2.84 (dd, $J=18.6,4.3 \mathrm{~Hz}$, $2 \mathrm{H}), 2.34(\mathrm{~s}, 3 \mathrm{H})$.

${ }^{13} \mathrm{C}$ NMR $\left(125 \mathrm{MHz}\right.$, DMSO- $\left.d_{6}\right): \delta=174.4,138.7,137.8,130.8,129.1$, 126.0, 122.6, 113.0, 40.9, 35.1, 21.4.

HRMS (ESI): $m / z[M+H]^{+}$calcd for $\mathrm{C}_{13} \mathrm{H}_{13} \mathrm{O}_{4}: 233.0814$; found: 233.0808 .

\section{6a-Methyldihydrofuro[2,3-b]furan-2,5(3H,6aH)-dione (14)}

Yield: $230 \mathrm{mg}$ (50\%); off-white solid; mp 93-95 ${ }^{\circ} \mathrm{C}$.

${ }^{1} \mathrm{H}$ NMR $\left(500 \mathrm{MHz}, \mathrm{CDCl}_{3}\right): \delta=3.12-3.06(\mathrm{~m}, 1 \mathrm{H}), 3.05-2.98(\mathrm{~m}, 2 \mathrm{H})$, 2.60-2.50 (m, $2 \mathrm{H}), 1.80$ (s, $3 \mathrm{H})$.

${ }^{13} \mathrm{C}$ NMR $\left(125 \mathrm{MHz}, \mathrm{CDCl}_{3}\right): \delta=172.6,113.1,38.9,35.4,23.8$.

HRMS (ESI): $m / z[M+H]^{+}$calcd for $\mathrm{C}_{7} \mathrm{H}_{9} \mathrm{O}_{4}:$ 157.0501; found: 157.0490 .

\section{6a-Ethyldihydrofuro[2,3-b]furan-2,5(3H,6aH)-dione (15)}

Yield: $210 \mathrm{mg}$ (42\%); off-white solid; mp $60-61{ }^{\circ} \mathrm{C}$.

${ }^{1} \mathrm{H}$ NMR $\left(500 \mathrm{MHz}, \mathrm{CDCl}_{3}\right): \delta=3.16-3.07(\mathrm{~m}, 1 \mathrm{H}), 2.96(\mathrm{dd}, J=18.6$, $9.6 \mathrm{~Hz}, 2 \mathrm{H}), 2.53(\mathrm{dd}, J=18.6,4.5 \mathrm{~Hz}, 2 \mathrm{H}), 2.01(\mathrm{q}, J=7.5 \mathrm{~Hz}, 2 \mathrm{H})$, $1.04(\mathrm{t}, J=7.6 \mathrm{~Hz}, 3 \mathrm{H})$.

${ }^{13} \mathrm{C}$ NMR $\left(125 \mathrm{MHz}, \mathrm{CDCl}_{3}\right): \delta=172.73,115.31,36.76,35.64,30.28$, 7.13 .

HRMS (ESI): $m / z[M+H]^{+}$calcd for $\mathrm{C}_{7} \mathrm{H}_{11} \mathrm{O}_{4}$ : 171.0657; found: 171.0651.

\section{6a-Isopropyldihydrofuro[2,3-b]furan-2,5(3H,6aH)-dione (16)}

Yield: $201 \mathrm{mg}$ (37\%); off-white solid; mp 88-89 ${ }^{\circ} \mathrm{C}$.

${ }^{1} \mathrm{H}$ NMR (500 MHz, $\mathrm{CDCl}_{3}$ ): $\delta=3.17(\mathrm{tt}, J=9.1,4.3 \mathrm{~Hz}, 1 \mathrm{H}$ ), 2.97 (dd, $J=18.7,10.0 \mathrm{~Hz}, 2 \mathrm{H}$ ), 2.54 (dd, $J=18.7,4.4 \mathrm{~Hz}, 2 \mathrm{H}$ ), 2.22 (hept, $J=$ $6.8 \mathrm{~Hz}, 1 \mathrm{H}), 1.06(\mathrm{~d}, J=6.9 \mathrm{~Hz}, 6 \mathrm{H})$.

${ }^{13} \mathrm{C}$ NMR $\left(125 \mathrm{MHz}, \mathrm{CDCl}_{3}\right.$ ): $\delta=172.7,117.2,36.2,35.1,34.9,16.0$. HRMS (ESI): $m / z[M+H]^{+}$calcd for $\mathrm{C}_{8} \mathrm{H}_{13} \mathrm{O}_{4}$ : 185.0814; found: 185.0802 .

\section{6a-Cyclohexyldihydrofuro[2,3-b]furan-2,5(3H,6aH)-dione (18)}

Yield: $145 \mathrm{mg}$ (22\%); off-white solid; mp 143-145 ${ }^{\circ} \mathrm{C}$.

${ }^{1} \mathrm{H}$ NMR $\left(500 \mathrm{MHz}, \mathrm{CDCl}_{3}\right): \delta=3.18(\mathrm{tt}, J=9.5,4.5 \mathrm{~Hz}, 1 \mathrm{H}), 2.95(\mathrm{dd}$, $J=18.6,10.0 \mathrm{~Hz}, 2 \mathrm{H}), 2.52$ (dd, $J=18.7,4.4 \mathrm{~Hz}, 2 \mathrm{H}), 1.96-1.67$ (m, $6 \mathrm{H}), 1.34-1.04$ (m, $5 \mathrm{H})$.

${ }^{13} \mathrm{C}$ NMR $\left(125 \mathrm{MHz}, \mathrm{CDCl}_{3}\right): \delta=172.67,116.68,44.52,36.06,34.96$, 25.97, 25.76, 25.40.

HRMS (ESI): $m / z[M+\mathrm{H}]^{+}$calcd for $\mathrm{C}_{12} \mathrm{H}_{17} \mathrm{O}_{4}$ : 225.1127; found: 225.1120 .

\section{Desymmetrization of Fittig Lactones; General Procedure}

All substrates were prepared in racemic form by using this procedure, except when 20 mol\% DMAP was used instead of chiral catalyst.

In a $25 \mathrm{~mL}$ one-necked flask, organocatalyst $3 d$ (68 mg, $0.1 \mathrm{mmol}, 0.1$ equiv) was suspended in anhydrous dioxane ( $5 \mathrm{~mL}, 0.2 \mathrm{M}$ for the substrate), and $N, N$-diethylacetamide ( $125 \mu \mathrm{L}, 1.0 \mathrm{mmol}, 1.0$ equiv) was added to the flask. The corresponding Fittig lactone $(1.0 \mathrm{mmol}, 1.0$ equiv) and alcohol (10 mmol, 10 equiv) were added sequentially and the mixture was stirred at r.t. Upon completion of the reaction as indicated by GC-MS or TLC (2-6 days), the solvent was evaporated and the residue was taken up in $\mathrm{CH}_{2} \mathrm{Cl}_{2}(15 \mathrm{~mL})$ and washed with $10 \%$ citric acid $(2 \times 5 \mathrm{~mL})$. The inorganic phase was extracted with $\mathrm{CH}_{2} \mathrm{Cl}_{2}$ $(3 \times 5 \mathrm{~mL})$, the combined organic phase was dried over $\mathrm{Na}_{2} \mathrm{SO}_{4}$, filtered and evaporated to dryness. Isolation by flash chromatography with a short column (hexanes/EtOAc, $1 \% \mathrm{AcOH}$ ) afforded the pure product. Determination of ee data is summarised in the section 'Derivatisation for chiral HPLC analysis'.

\section{3-Benzoyl-5-methoxy-5-oxopentanoic Acid (2)}

Yield: $182 \mathrm{mg}$ (73\%); white solid; ee: 92\%; $t_{R}=13.3$ (minor), 23.5 (major) $\mathrm{min}$ (Chiralpak AD; $50 \%$ hexane $/ 50 \% \mathrm{IPA} ; 1 \mathrm{~mL} / \mathrm{min} ; 20{ }^{\circ} \mathrm{C} ; \lambda=$ $247 \mathrm{~nm} ; 20 \mu \mathrm{L})$.

${ }^{1} \mathrm{H}$ NMR $\left(500 \mathrm{MHz}, \mathrm{CDCl}_{3}\right): \delta=8.02-7.92(\mathrm{~m}, 2 \mathrm{H}), 7.57(\mathrm{t}, J=7.6 \mathrm{~Hz}$, $1 \mathrm{H}), 7.47(\mathrm{t}, J=7.7 \mathrm{~Hz}, 2 \mathrm{H}), 4.28$ (quint., $J=6.9 \mathrm{~Hz}, 1 \mathrm{H}), 3.63(\mathrm{~d}, J=$ $2.1 \mathrm{~Hz}, 3 \mathrm{H}$ ), 2.86 (ddd, $J=29.0,16.8,7.0 \mathrm{~Hz}, 2 \mathrm{H}$ ), 2.54 (td, $J=16.5$, $6.8 \mathrm{~Hz}, 2 \mathrm{H})$.

${ }^{13} \mathrm{C}$ NMR $\left(125 \mathrm{MHz}, \mathrm{CDCl}_{3}\right): \delta=200.5,177.3,171.8,135.4,133.4$, 128.8, 128.5, 51.9, 38.6, 35.6, 35.4.

HRMS (ESI): $m / z[M+H]^{+}$calcd for $\mathrm{C}_{13} \mathrm{H}_{15} \mathrm{O}_{5}$ : 251.0919; found: 251.0903 .

\section{5-Methoxy-5-oxo-3-[4-(trifluoromethyl)benzoyl]pentanoic Acid (20)}

Yield: $260 \mathrm{mg}$ (82\%); colourless oil; ee: $84 \% ; t_{R}=9.0$ (major), 10.0 (minor) $\mathrm{min}$ (Chiralpak IB; $85 \%$ hexane $/ 15 \% \mathrm{EtOH} ; 1 \mathrm{~mL} / \mathrm{min} ; 20{ }^{\circ} \mathrm{C} ; \lambda=$ $247 \mathrm{~nm} ; 20 \mu \mathrm{L})$.

${ }^{1} \mathrm{H}$ NMR $\left(500 \mathrm{MHz}, \mathrm{CDCl}_{3}\right): \delta=8.08(\mathrm{~d}, J=8.1 \mathrm{~Hz}, 2 \mathrm{H}), 7.74(\mathrm{~d}, J=$ $8.2 \mathrm{~Hz}, 2 \mathrm{H}$ ), 4.25 (quint., $J=6.8 \mathrm{~Hz}, 1 \mathrm{H}$ ), $3.63(\mathrm{~s}, 3 \mathrm{H}$ ), 2.85 (ddd, $J=$ $34.3,17.0,7.3 \mathrm{~Hz}, 2 \mathrm{H}$ ), 2.57 (ddd, $J=21.4,16.9,6.6 \mathrm{~Hz}, 2 \mathrm{H}$ ).

${ }^{13} \mathrm{C}$ NMR $\left(125 \mathrm{MHz}, \mathrm{CDCl}_{3}\right): \delta=199.9,176.9,171.6,138.4,134.6(\mathrm{q}, J=$ $32.7 \mathrm{~Hz}), 128.9,125.8(\mathrm{q}, J=3.8 \mathrm{~Hz}), 123.5(\mathrm{q}, J=272.8 \mathrm{~Hz}), 52.1,38.8$, 35.6, 35.3.

HRMS (ESI): $m / z$ [M $+\mathrm{H}]^{+}$calcd for $\mathrm{C}_{14} \mathrm{H}_{14} \mathrm{~F}_{3} \mathrm{O}_{5}$ : 319.0793; found: 319.0786 .

\section{5-Methoxy-3-(4-methoxybenzoyl)-5-oxopentanoic Acid (21)}

Yield: $202 \mathrm{mg}$ (72\%); colourless oil; ee: $92 \% ; t_{R}=13.0$ (major), 15.5 (minor) min (Chiralpak IB; 85\% hexane/15\% EtOH; $1 \mathrm{~mL} / \mathrm{min} ; 20{ }^{\circ} \mathrm{C} ; \lambda$ $=271 \mathrm{~nm} ; 20 \mu \mathrm{L})$.

${ }^{1} \mathrm{H}$ NMR $\left(500 \mathrm{MHz}, \mathrm{CDCl}_{3}\right): \delta=7.98(\mathrm{~d}, J=8.7 \mathrm{~Hz}, 2 \mathrm{H}), 6.95(\mathrm{~d}, J=$ $8.8 \mathrm{~Hz}, 2 \mathrm{H}$ ), 4.25 (quint., $J=7.0 \mathrm{~Hz}, 1 \mathrm{H}), 3.87(\mathrm{~s}, 3 \mathrm{H}), 3.64(\mathrm{~s}, 3 \mathrm{H})$, 2.85 (ddd, $J=28.2,16.7,6.9 \mathrm{~Hz}, 2 \mathrm{H}), 2.54(\mathrm{td}, J=16.4,6.9 \mathrm{~Hz}, 2 \mathrm{H})$.

${ }^{13} \mathrm{C}$ NMR $\left(125 \mathrm{MHz}, \mathrm{CDCl}_{3}\right): \delta=198.9,176.7,171.9,163.9,130.9$, $128.3,114.0,55.5,51.9,38.2,35.8,35.5$.

HRMS (ESI): $m / z[M+H]^{+}$calcd for $\mathrm{C}_{14} \mathrm{H}_{17} \mathrm{O}_{6}: 281.1025$; found: 281.1016 . 


\section{3-(4-Chlorobenzoyl)-5-methoxy-5-oxopentanoic Acid (22)}

Yield: $241 \mathrm{mg}$ (85\%); colourless oil; ee: $93 \% ; t_{R}=9.0$ (major), 10.0 (minor) $\mathrm{min}$ (Chiralpak IB; $85 \%$ hexane $/ 15 \% \mathrm{EtOH} ; 1 \mathrm{~mL} / \mathrm{min} ; 20{ }^{\circ} \mathrm{C} ; \lambda=$ $256 \mathrm{~nm} ; 20 \mu \mathrm{L})$.

${ }^{1} \mathrm{H}$ NMR $\left(500 \mathrm{MHz}, \mathrm{CDCl}_{3}\right): \delta=7.91(\mathrm{~d}, J=8.7 \mathrm{~Hz}, 2 \mathrm{H}), 7.43(\mathrm{~d}, J=$ $8.6 \mathrm{~Hz}, 2 \mathrm{H}$ ), 4.20 (quint., $J=6.9 \mathrm{~Hz}, 1 \mathrm{H}$ ), $3.61(\mathrm{~s}, 3 \mathrm{H}$ ), 2.82 (ddd, $J=$ $31.5,16.8,7.2 \mathrm{~Hz}, 2 \mathrm{H}$ ), 2.53 (td, $J=17.2,6.6 \mathrm{~Hz}, 2 \mathrm{H}$ ).

${ }^{13} \mathrm{C}$ NMR $\left(125 \mathrm{MHz}, \mathrm{CDCl}_{3}\right): \delta=199.5,177.1,171.7,139.9,133.9$, $130.0,129.1,52.0,38.5,35.7,35.4$.

HRMS (ESI): $m / z[\mathrm{M}+\mathrm{H}]^{+}$calcd for $\mathrm{C}_{13} \mathrm{H}_{14} \mathrm{ClO}_{5}$ : 285.0530; found: 285.0522 .

\section{3-(2-Fluorobenzoyl)-5-methoxy-5-oxopentanoic Acid (23)}

Yield: $225 \mathrm{mg}$ (84\%); colourless oil; ee: $75 \% ; t_{R}=12.3$ (minor), 17.3 (major) $\mathrm{min}$ (Chiralpak AD; 50\% hexane $/ 50 \%$ IPA; $1 \mathrm{~mL} / \mathrm{min} ; 20{ }^{\circ} \mathrm{C} ; \lambda=$ $239 \mathrm{~nm} ; 20 \mu \mathrm{L})$.

${ }^{1} \mathrm{H}$ NMR $\left(500 \mathrm{MHz}, \mathrm{CDCl}_{3}\right): \delta=7.81(\mathrm{td}, J=7.6,1.8 \mathrm{~Hz}, 1 \mathrm{H}), 7.51$ (dddd, $J=8.6,7.1,5.0,1.8 \mathrm{~Hz}, 1 \mathrm{H}), 7.22(\mathrm{td}, J=7.6,1.1 \mathrm{~Hz}, 1 \mathrm{H}), 7.12$ (ddd, $J=11.2,8.3,1.0 \mathrm{~Hz}, 1 \mathrm{H}$ ), 4.08 (quint., $J=6.7 \mathrm{~Hz}, 1 \mathrm{H}$ ), 3.63 (s, $3 \mathrm{H}$ ), 2.88 (dddd, $J=22.5,16.7,6.8,1.4 \mathrm{~Hz}, 2 \mathrm{H}$ ), 2.63-2.48 (m, $2 \mathrm{H}$ ).

${ }^{13} \mathrm{C}$ NMR $\left(125 \mathrm{MHz}, \mathrm{CDCl}_{3}\right): \delta=198.6,177.2,171.8,161.2$ (d, $J=$ $254.1 \mathrm{~Hz}), 134.7(\mathrm{~d}, J=9.2 \mathrm{~Hz}), 131.2(\mathrm{~d}, J=2.4 \mathrm{~Hz}), 124.7(\mathrm{~d}, J=$ $3.2 \mathrm{~Hz}), 124.7(\mathrm{~d}, J=13.9 \mathrm{~Hz}), 116.6(\mathrm{~d}, J=23.9 \mathrm{~Hz}), 51.9,43.2(\mathrm{~d}, J=$ $6.6 \mathrm{~Hz}), 34.8(\mathrm{~d}, J=16.7 \mathrm{~Hz})$.

HRMS (ESI): $m / z[M+H]^{+}$calcd for $\mathrm{C}_{13} \mathrm{H}_{14} \mathrm{FO}_{5}$ : 269.0825; found: 269.0817.

\section{3-(2-Chlorobenzoyl)-5-methoxy-5-oxopentanoic Acid (24)}

Yield: $219 \mathrm{mg}$ (77\%); colourless oil; ee: $49 \% ; t_{R}=12.2$ (minor), 23.5 (major) min (Chiralpak AD; $50 \%$ hexane $/ 50 \%$ IPA; $1 \mathrm{~mL} / \mathrm{min} ; 20{ }^{\circ} \mathrm{C} ; \lambda=$ $263 \mathrm{~nm} ; 20 \mu \mathrm{L})$.

${ }^{1} \mathrm{H}$ NMR $\left(500 \mathrm{MHz}, \mathrm{CDCl}_{3}\right): \delta=10.15(\mathrm{~s}, 1 \mathrm{H}), 7.69-7.51(\mathrm{~m}, 1 \mathrm{H})$, 7.42-7.30 (m, $3 \mathrm{H}), 4.06$ (quint., $J=6.8,6.0 \mathrm{~Hz}, 1 \mathrm{H}), 3.64(\mathrm{~s}, 3 \mathrm{H})$, 2.94-2.79 (m, $2 \mathrm{H}), 2.66-2.53(\mathrm{~m}, 2 \mathrm{H})$.

${ }^{13} \mathrm{C}$ NMR $\left(125 \mathrm{MHz}, \mathrm{CDCl}_{3}\right): \delta=202.0,177.0,171.7,137.7,131.9$, $131.1,130.5,129.5,127.0,52.0,43.1,34.3,34.2$.

HRMS (ESI): $m / z[\mathrm{M}+\mathrm{H}]^{+}$calcd for $\mathrm{C}_{13} \mathrm{H}_{14} \mathrm{ClO}_{5}$ : 285.0530; found: 285.0521.

\section{3-(2-Bromobenzoyl)-5-methoxy-5-oxopentanoic Acid (25)}

Yield: $183 \mathrm{mg}$ (56\%); colourless oil; ee: $37 \% ; t_{R}=13.1$ (minor), 25.5 (major) $\mathrm{min}$ (Chiralpak AD; 50\% hexane $/ 50 \%$ IPA; $1 \mathrm{~mL} / \mathrm{min} ; 20{ }^{\circ} \mathrm{C} ; \lambda=$ $247 \mathrm{~nm} ; 20 \mu \mathrm{L})$.

${ }^{1} \mathrm{H} \mathrm{NMR}\left(500 \mathrm{MHz}, \mathrm{CDCl}_{3}\right): \delta=10.19(\mathrm{~s}, 1 \mathrm{H}), 7.59$ (ddd, $J=7.6,5.1$, $1.4 \mathrm{~Hz}, 2 \mathrm{H}), 7.37(\mathrm{td}, J=7.5,1.1 \mathrm{~Hz}, 1 \mathrm{H}), 7.29(\mathrm{td}, J=7.7,1.7 \mathrm{~Hz}, 1 \mathrm{H})$, 4.05 (quint., $J=6.7 \mathrm{~Hz}, 1 \mathrm{H}$ ), $3.63(\mathrm{~s}, 3 \mathrm{H}), 2.85$ (ddd, $J=31.8,17.0$, $6.6 \mathrm{~Hz}, 2 \mathrm{H}), 2.58$ (ddd, $J=18.8,17.0,6.8 \mathrm{~Hz}, 2 \mathrm{H}$ ).

${ }^{13} \mathrm{C}$ NMR $\left(125 \mathrm{MHz}, \mathrm{CDCl}_{3}\right): \delta=202.6,177.2,171.7,139.7,133.8$, $131.9,129.3,127.4,119.3,52.0,43.0,34.2,34.2$.

HRMS (ESI): $m / z$ [M - H] $]^{-}$calcd for $\mathrm{C}_{13} \mathrm{H}_{12} \mathrm{BrO}_{5}$ : 326.9868; found: 326.9874

\section{5-Methoxy-3-(2-methylbenzoyl)-5-oxopentanoic Acid (26)}

Yield: $201 \mathrm{mg}$ (76\%); colourless oil; ee: 74\%; $t_{R}=10.4$ (minor), 23.2 (major) $\mathrm{min}$ (Chiralpak AD; 50\% hexane $/ 50 \% \mathrm{IPA} ; 1 \mathrm{~mL} / \mathrm{min} ; 20{ }^{\circ} \mathrm{C} ; \lambda=$ $247 \mathrm{~nm} ; 20 \mu \mathrm{L})$.
${ }^{1} \mathrm{H}$ NMR $\left(500 \mathrm{MHz}, \mathrm{CDCl}_{3}\right): \delta=7.74-7.69(\mathrm{~m}, 1 \mathrm{H}), 7.40-7.34(\mathrm{~m}, 1 \mathrm{H})$, 7.29-7.22 (m, $2 \mathrm{H}), 4.09$ (quint., $J=6.8 \mathrm{~Hz}, 1 \mathrm{H}), 3.62(\mathrm{~s}, 3 \mathrm{H}), 2.81$ (ddd, $J=33.8,16.8,6.9 \mathrm{~Hz}, 2 \mathrm{H}$ ), 2.54 (ddd, $J=19.7,16.8,6.6 \mathrm{~Hz}, 2 \mathrm{H}$ ), $2.42(\mathrm{~s}, 3 \mathrm{H})$.

${ }^{13} \mathrm{C}$ NMR $\left(125 \mathrm{MHz}, \mathrm{CDCl}_{3}\right): \delta=203.7,177.3,171.8,138.6,136.6$, 131.8, 131.4, 128.3, 125.8, 51.9, 41.8, 34.9, 34.8, 20.6.

HRMS (ESI): $m / z[M+\mathrm{H}]^{+}$calcd for $\mathrm{C}_{14} \mathrm{H}_{17} \mathrm{ClO}_{5}$ : 265.1076; found: 265.1068 .

\section{3-(3-Fluorobenzoyl)-5-methoxy-5-oxopentanoic Acid (27)}

Yield: $206 \mathrm{mg}$ (77\%); colourless oil; ee: $89 \% ; t_{R}=18.5$ (minor), 23.5 (major) $\min$ (Chiralpak AD; $50 \%$ hexane $/ 50 \% \mathrm{IPA} ; 1 \mathrm{~mL} / \mathrm{min} ; 20{ }^{\circ} \mathrm{C} ; \lambda=$ $244 \mathrm{~nm} ; 20 \mu \mathrm{L})$.

${ }^{1} \mathrm{H}$ NMR $\left(500 \mathrm{MHz}, \mathrm{CDCl}_{3}\right): \delta=10.28(\mathrm{~s}, 1 \mathrm{H}), 7.73(\mathrm{~d}, J=7.8 \mathrm{~Hz}, 1 \mathrm{H})$, $7.62(\mathrm{~d}, J=9.4 \mathrm{~Hz}, 1 \mathrm{H}), 7.42(\mathrm{td}, J=7.9,5.3 \mathrm{~Hz}, 1 \mathrm{H}), 7.24$ (ddd, $J=$ 10.8, 6.6, $2.5 \mathrm{~Hz}, 1 \mathrm{H}$ ), 4.17 (t, J = 6.9 Hz, $1 \mathrm{H}$ ), 3.59 (s, $3 \mathrm{H}$ ), 2.80 (ddd, $J=16.6,12.9,6.9 \mathrm{~Hz}, 2 \mathrm{H}), 2.51$ (dd, $J=16.9,6.6 \mathrm{~Hz}, 2 \mathrm{H}$ ).

${ }^{13} \mathrm{C}$ NMR $\left(125 \mathrm{MHz}, \mathrm{CDCl}_{3}\right): \delta=199.7,177.0,171.8,162.9(\mathrm{~d}, J=$ $247.9 \mathrm{~Hz}), 137.7(\mathrm{~d}, J=6.2 \mathrm{~Hz}), 130.4(\mathrm{~d}, J=7.6 \mathrm{~Hz}), 124.2(\mathrm{~d}, J=$ $3.1 \mathrm{~Hz}), 120.4(\mathrm{~d}, J=21.4 \mathrm{~Hz}), 115.3(\mathrm{~d}, J=22.5 \mathrm{~Hz}), 52.0,38.9,35.6$, 35.5.

HRMS (ESI): $m / z[M+H]^{+}$calcd for $\mathrm{C}_{13} \mathrm{H}_{14} \mathrm{FO}_{5}$ : 269.0825; found: 269.08177.

\section{3-(3-Chlorobenzoyl)-5-methoxy-5-oxopentanoic Acid (28)}

Yield: $170 \mathrm{mg}$ (60\%); colourless oil; ee: $94 \% ; t_{R}=19.5$ (minor), 25.5 (major) $\mathrm{min}$ (Chiralpak AD; 50\% hexane $/ 50 \% \mathrm{IPA} ; 1 \mathrm{~mL} / \mathrm{min} ; 20{ }^{\circ} \mathrm{C} ; \lambda=$ $243 \mathrm{~nm} ; 20 \mu \mathrm{L})$.

${ }^{1} \mathrm{H}$ NMR $\left(500 \mathrm{MHz}, \mathrm{CDCl}_{3}\right): \delta=7.93(\mathrm{~s}, 1 \mathrm{H}), 7.85(\mathrm{dt}, J=7.8,1.4 \mathrm{~Hz}$, $1 \mathrm{H}$ ), 7.61-7.50 (m, $1 \mathrm{H}), 7.41(\mathrm{t}, J=7.9 \mathrm{~Hz}, 1 \mathrm{H}), 4.20$ (quint., $J=$ $7.0 \mathrm{~Hz}, 1 \mathrm{H}$ ), 3.63 (s, $3 \mathrm{H}$ ), 2.83 (ddd, $J=28.7,16.9,7.1 \mathrm{~Hz}, 2 \mathrm{H}), 2.64-$ $2.48(\mathrm{~m}, 2 \mathrm{H})$.

${ }^{13} \mathrm{C}$ NMR $\left(125 \mathrm{MHz}, \mathrm{CDCl}_{3}\right): \delta=199.5,176.9,171.6,137.2,135.2$, 133.3, 130.1, 128.6, 126.6, 52.0, 38.7, 35.6, 35.4.

HRMS (ESI): $m / z[\mathrm{M}+\mathrm{H}]^{+}$calcd for $\mathrm{C}_{13} \mathrm{H}_{14} \mathrm{ClO}_{5}$ : 285.0530; found: 285.0522 .

\section{5-Methoxy-3-(3-methylbenzoyl)-5-oxopentanoic Acid (29)}

Yield: $200 \mathrm{mg}$ (76\%); colourless oil; ee: $91 \% ; t_{R}=18.5$ (minor), 25.5 (major) min (Chiralpak AD; 50\% hexane $/ 50 \% \mathrm{IPA} ; 1 \mathrm{~mL} / \mathrm{min} ; 20{ }^{\circ} \mathrm{C} ; \lambda=$ $254 \mathrm{~nm} ; 20 \mu \mathrm{L})$.

${ }^{1} \mathrm{H}$ NMR $\left(500 \mathrm{MHz}, \mathrm{CDCl}_{3}\right): \delta=7.78(\mathrm{~d}, J=6.2 \mathrm{~Hz}, 2 \mathrm{H}), 7.45-7.32(\mathrm{~m}$, $2 \mathrm{H}$ ), 4.27 (quint., $J=7.0 \mathrm{~Hz}, 1 \mathrm{H}$ ), $3.64(\mathrm{~s}, 3 \mathrm{H}$ ), 2.86 (ddd, $J=27.5$, $16.8,6.9 \mathrm{~Hz}, 2 \mathrm{H}), 2.54$ (td, $J=16.3,6.9 \mathrm{~Hz}, 2 \mathrm{H}), 2.41(\mathrm{~s}, 3 \mathrm{H})$.

${ }^{13} \mathrm{C}$ NMR $\left(125 \mathrm{MHz}, \mathrm{CDCl}_{3}\right): \delta=200.6,177.0,171.8,138.7,135.4$, 134.2, 129.0, 128.7, 125.7, 51.9, 38.7, 35.6, 35.3, 21.3.

HRMS (ESI): $m / z[\mathrm{M}+\mathrm{H}]^{+}$calcd for $\mathrm{C}_{14} \mathrm{H}_{17} \mathrm{O}_{5}$ : 265.1076; found: 265.1069 .

\section{3-Acetyl-5-methoxy-5-oxopentanoic Acid (30)}

Yield: $112 \mathrm{mg}$ (60\%); white crystals; decomposition at $89-96{ }^{\circ} \mathrm{C}$; ee: 78\%; $t_{R}=51.0$ (minor), 57.5 (major) min (Chiralpak OJ; 70\% hexane $\left./ 30 \% \mathrm{IPA} ; 1 \mathrm{~mL} / \mathrm{min} ; 2{ }^{\circ} \mathrm{C} ; \lambda=265 \mathrm{~nm} ; 20 \mu \mathrm{L}\right)$.

${ }^{1} \mathrm{H} \mathrm{NMR}\left(500 \mathrm{MHz}\right.$, DMSO- $\left.d_{6}\right): \delta=3.56(\mathrm{~s}, 3 \mathrm{H}), 3.12(\mathrm{t}, J=6.8 \mathrm{~Hz}, 1 \mathrm{H})$, 2.60 (ddd, $J=30.6,16.8,7.5 \mathrm{~Hz}, 2 \mathrm{H}$ ), 2.42 (ddd, $J=25.6,16.8,5.9 \mathrm{~Hz}$, $2 \mathrm{H}), 2.15$ (s, $3 \mathrm{H})$. 
${ }^{13} \mathrm{C}$ NMR $\left(125 \mathrm{MHz}\right.$, DMSO- $\left.d_{6}\right): \delta=209.0,173.2,172.3,51.9,44.3$, 35.1, 34.7, 28.9 .

HRMS (ESI): $m / z[M+H]^{+}$calcd for $\mathrm{C}_{8} \mathrm{H}_{13} \mathrm{O}_{5}: 189.0763$; found: 189.0756 .

\section{3-(2-Methoxy-2-oxoethyl)-4-oxohexanoic Acid (31)}

Yield: $115 \mathrm{mg}$ (57\%); colourless oil; ee: $88 \% ; t_{R}=29.5$ (minor), 36.5 (major) min (Chiralpak OJ; $70 \%$ hexane $/ 30 \% \mathrm{IPA} ; 1 \mathrm{~mL} / \mathrm{min} ; 20{ }^{\circ} \mathrm{C} ; \lambda=$ $265 \mathrm{~nm} ; 20 \mu \mathrm{L})$.

${ }^{1} \mathrm{H}$ NMR (500 MHz, $\mathrm{CDCl}_{3}$ ): $\delta=3.64(\mathrm{~s}, 3 \mathrm{H}$ ), 3.29 (quint., $J=6.8 \mathrm{~Hz}$, $1 \mathrm{H}$ ), 2.70 (ddd, $J=23.6,16.9,7.6 \mathrm{~Hz}, 2 \mathrm{H}$ ), 2.58 (q, $J=7.0 \mathrm{~Hz}, 2 \mathrm{H}$ ), 2.45 (ddd, $J=16.8,11.7,6.2 \mathrm{~Hz}, 2 \mathrm{H}), 1.02(\mathrm{t}, J=7.2 \mathrm{~Hz}, 3 \mathrm{H}$ ).

${ }^{13} \mathrm{C}$ NMR $\left(125 \mathrm{MHz}, \mathrm{CDCl}_{3}\right): \delta=211.5,177.1,171.9,52.0,42.8,35.2$, 35.1, 35.0, 7.5.

HRMS (ESI): $m / z[M+H]^{+}$calcd for $\mathrm{C}_{9} \mathrm{H}_{15} \mathrm{O}_{5}:$ 203.0919; found: 203.0912 .

\section{3-(2-Methoxy-2-oxoethyl)-5-methyl-4-oxohexanoic Acid (32)}

Yield: $116 \mathrm{mg}$ (54\%); colourless oil; ee: $88 \% ; t_{R}=36.5$ (minor), 40.5 (major) min (Chiralpak OJ; $85 \%$ hexane $/ 15 \%$ IPA; $1 \mathrm{~mL} / \mathrm{min} ; 20{ }^{\circ} \mathrm{C} ; \lambda=$ $264 \mathrm{~nm} ; 20 \mu \mathrm{L})$.

${ }^{1} \mathrm{H}$ NMR (500 MHz, $\mathrm{CDCl}_{3}$ ): $\delta=3.65$ (s, $3 \mathrm{H}$ ), 3.48 (quint., $J=6.6 \mathrm{~Hz}$, $1 \mathrm{H}), 2.83$ (hept, $J=7.0,5.8 \mathrm{~Hz}, 1 \mathrm{H}$ ), 2.71 (ddd, $J=23.2,16.8,6.9 \mathrm{~Hz}$, $2 \mathrm{H}$ ), 2.43 (ddd, $J=16.8,11.4,6.7 \mathrm{~Hz}, 2 \mathrm{H}$ ), 1.10 (d, $J=6.9 \mathrm{~Hz}, 6 \mathrm{H}$ ).

${ }^{13} \mathrm{C}$ NMR $\left(125 \mathrm{MHz}, \mathrm{CDCl}_{3}\right): \delta=214.2,177.2,171.8,51.9,41.7,39.4$, 35.0, 34.9, 18.4, 18.3 .

HRMS (ESI): $m / z[M+H]^{+}$calcd for $\mathrm{C}_{10} \mathrm{H}_{17} \mathrm{O}_{5}: 217.1076$; found: 217.1068.

\section{3-(Cyclohexanecarbonyl)-5-methoxy-5-oxopentanoic Acid (33)}

Yield: $150 \mathrm{mg}$ (59\%); colourless oil; ee: $92 \% ; t_{R}=22.5$ (minor), 26.5 (major) min (Chiralpak OJ; $85 \%$ hexane/15\% IPA; $1 \mathrm{~mL} / \mathrm{min} ; 20{ }^{\circ} \mathrm{C} ; \lambda=$ $265 \mathrm{~nm} ; 20 \mu \mathrm{L})$.

${ }^{1} \mathrm{H}$ NMR (500 MHz, $\mathrm{CDCl}_{3}$ ): $\delta=3.67$ (s, $3 \mathrm{H}$ ), 3.52-3.45 (m, $1 \mathrm{H}$ ), 2.72 (ddd, $J=20.5,16.8,6.9 \mathrm{~Hz}, 2 \mathrm{H}$ ), 2.57 (tt, $J=11.0,3.0 \mathrm{~Hz}, 1 \mathrm{H}$ ), 2.43 (ddd, $J=16.8,11.0,6.9 \mathrm{~Hz}, 2 \mathrm{H}), 1.94-1.09(\mathrm{~m}, 10 \mathrm{H})$.

${ }^{13} \mathrm{C}$ NMR $\left(125 \mathrm{MHz}, \mathrm{CDCl}_{3}\right): \delta=213.3,177.1,171.8,51.9,49.5,41.7$, 34.9, 34.8, 28.7, 28.5, 25.7, 25.7, 25.6.

HRMS (ESI): $m / z[M-H]^{-}$calcd for $\mathrm{C}_{13} \mathrm{H}_{19} \mathrm{O}_{5}$ : 255.1232; found: 255.1235 .

\section{3-Benzoyl-5-ethoxy-5-oxopentanoic Acid (34)}

Yield: $237 \mathrm{mg}$ (90\%); colourless oil; ee: $95 \% ; t_{R}=13.0$ (minor), 22.6 (major) $\mathrm{min}$ (Chiralpak AD; 50\% hexane $/ 50 \% \mathrm{IPA} ; 1 \mathrm{~mL} / \mathrm{min} ; 20{ }^{\circ} \mathrm{C} ; \lambda=$ $247 \mathrm{~nm} ; 20 \mu \mathrm{L})$.

${ }^{1} \mathrm{H}$ NMR $\left(500 \mathrm{MHz}, \mathrm{CDCl}_{3}\right): \delta=10.09(\mathrm{~s}, 1 \mathrm{H}), 7.97(\mathrm{dt}, J=7.2,1.3 \mathrm{~Hz}$, $2 \mathrm{H}$ ), 7.61-7.52 (m, $1 \mathrm{H}), 7.46$ (dd, $J=8.4,7.1 \mathrm{~Hz}, 2 \mathrm{H}), 4.27$ (quint., $J=$ $6.9 \mathrm{~Hz}, 1 \mathrm{H}$ ), 4.07 (qd, $J=7.1,2.5 \mathrm{~Hz}, 2 \mathrm{H}$ ), 2.84 (ddd, $J=33.8,16.7$, $7.1 \mathrm{~Hz}, 2 \mathrm{H}$ ), 2.53 (ddd, $J=19.2,16.7,6.8 \mathrm{~Hz}, 2 \mathrm{H}$ ), 1.17 (t, $J=7.2 \mathrm{~Hz}$, $3 \mathrm{H})$.

${ }^{13} \mathrm{C}$ NMR $\left(125 \mathrm{MHz}, \mathrm{CDCl}_{3}\right): \delta=200.6,177.2,171.3,135.5,133.4$, $128.8,128.5,61.0,38.6,35.9,35.4,14.0$.

HRMS (ESI): $m / z[M+H]^{+}$calcd for $\mathrm{C}_{14} \mathrm{H}_{16} \mathrm{O}_{5}$ : 265.1076; found: 265.1071 .

\section{3-Benzoyl-5-(benzyloxy)-5-oxopentanoic Acid (38)}

Yield: $303 \mathrm{mg}$ (93\%); colourless oil; ee: $95 \% ; t_{R}=18.8$ (minor), 26.9 (major) $\min$ (Chiralpak AD; 50\% hexane $/ 50 \% \mathrm{IPA} ; 1 \mathrm{~mL} / \mathrm{min} ; 20{ }^{\circ} \mathrm{C} ; \lambda=$ $247 \mathrm{~nm} ; 20 \mu \mathrm{L})$.

${ }^{1} \mathrm{H}$ NMR $\left(500 \mathrm{MHz}, \mathrm{CDCl}_{3}\right): \delta=10.29(\mathrm{~s}, 1 \mathrm{H}), 8.01-7.95(\mathrm{~m}, 2 \mathrm{H})$, 7.60-7.53 (m, $1 \mathrm{H}), 7.46$ (t, J = 7.8 Hz, $2 \mathrm{H}), 7.37-7.24(\mathrm{~m}, 5 \mathrm{H}), 5.12-$ $5.03(\mathrm{~m}, 2 \mathrm{H}), 4.31$ (quint., $J=6.9 \mathrm{~Hz}, 1 \mathrm{H}), 2.89$ (ddd, $J=17.0,7.1$, $5.1 \mathrm{~Hz}, 2 \mathrm{H}$ ), 2.58 (ddd, $J=17.8,16.8,6.8 \mathrm{~Hz}, 2 \mathrm{H}$ ).

${ }^{13} \mathrm{C}$ NMR $\left(125 \mathrm{MHz}, \mathrm{CDCl}_{3}\right): \delta=200.5,177.2,171.2,135.5,133.4$, $128.80,128.58,128.56,128.33,128.25,66.8,38.7,35.8,35.4$.

HRMS (ESI): $m / z[M+H]^{+}$calcd for $\mathrm{C}_{19} \mathrm{H}_{18} \mathrm{O}_{5}$ : 327.1232; found: 327.1226 .

\section{Derivatisation for Chiral HPLC Analysis}

In a screw-capped $4 \mathrm{~mL}$ vial, 4-dimethylaminopyridine (3 $\mathrm{mg}$, $0.025 \mathrm{mmol}, 0.05$ equiv), the corresponding 5 -methoxy-5-oxopentanoic acid ( $0.5 \mathrm{mmol}, 1.0$ equiv) and 2-nitrophenol ( $56 \mathrm{mg}, 0.45 \mathrm{mmol}$, 0.9 equiv) were added sequentially. $\mathrm{CH}_{2} \mathrm{Cl}_{2}$ was added as solvent (2.5 $\mathrm{mL}, 0.2 \mathrm{M}$ ) and then DCC (63 mg, $0.45 \mathrm{mmol}, 0.9$ equiv) was added in one portion. The resulting yellow solution turned cloudy in a couple of minutes due to the formation of $N, N^{\prime}$-dicyclohexylurea. The suspension was stirred at r.t. overnight. The product was separated by TLC then dissolved from the plate with $200 \mu \mathrm{L}$ HPLC ethanol, diluted to $1 \mathrm{~mL}$ with HPLC hexane and introduced to the HPLC system.

\section{Funding Information}

We are grateful for the financial support from NKFIH (K116150).

\section{Acknowledgment}

The authors thank Gitta Schlosser for HRMS measurements.

\section{References}

(1) (a) Borissov, A.; Davies, T. Q.; Ellis, S. R.; Fleming, T. A.; Richardson, M. S. W.; Dixon, D. J. Chem. Soc. Rev. 2016, 45, 5474. (b) Suzuki, T. Tetrahedron Lett. 2017, 58, 4731.

(2) (a) For a recent review, see: Merad, J.; Candy, M.; Pons, J.-M.; Bressy, C. Synthesis 2017, 49, 1938. Selected examples: (b) Phillips, E. M.; Roberts, J. M.; Scheidt, K. A. Org. Lett. 2010, 12, 2830. (c) Higuchi, K.; Suzuki, S.; Ueda, R.; Oshima, N.; Kobayashi, E.; Tayu, M.; Kawasaki, T. Org. Lett. 2015, 17, 154. (d) Park, K. H.; Chen, D. Y.-K. Chem. Commun. 2018, 13018. (e) Park, J.; Chen, D. Y.-K. Angew. Chem. Int. Ed. 2018, 57, 16152.

(3) (a) Chen, Y.; McDaid, P.; Deng, L. Chem. Rev. 2003, 103, 2965. (b) de Villegas, M. D.; Gálvez, J. A.; Etayo, P.; Badorrey, R.; LópezRam-de-Víu, P. Chem. Soc. Rev. 2011, 40, 5564.

(4) Gualtierotti, J.-B.; Pasche, D.; Wang, Q.; Zhu, J. Angew. Chem. Int. Ed. 2014, 53, 9926.

(5) (a) Shim, S. H.; Gloer, J. B.; Wicklow, D. T. J. Nat. Prod. 2006, 69, 1601. (b) Chen, X.-L.; Liu, H.-L.; Li, J.; Xin, G.-R.; Guo, Y.-W. Org. Lett. 2011, 13, 5032. (c) Weinaes, K.; Bahr, W. Justus Liebigs Ann. Chem. 1969, 724, 214.

(6) (a) Biber, A.; Koch, E. Planta Med. 1999, 65, 192. (b) Rossi, R.; Basilico, F.; Rossoni, G.; Riva, A.; Morazzoni, P.; Mauri, P. L. J. Pharm. Biomed. Anal. 2009, 50, 224. (c) Woelkart, K.; Feizlmayr, E.; Dittrich, P.; Beubler, E.; Pinl, F.; Suter, A.; Bauer, R. Phytother. Res. 2010, 24, 445. 
(7) (a) Acylals are cleaved by esterases and used in drug design: Gisch, N.; Balzarini, J.; Meier, C. J. Med. Chem. 2007, 50, 1658. (b) For a review on aldehyde acylals, see: Sydnes, L. K.; Sandberg, M. Proc. Indian Natn. Sci. Acad. Part A 2002, 68, 141.

(8) Fittig, R. Justus Liebigs Ann. Chem. 1901, 314, 1.

(9) (a) Ohsawa, S.; Morino, K.; Sudo, A.; Endo, T. Macromolecules 2011, 44, 1814. (b) Yamasaki, R.; Sudo, A.; Endo, T. J. Polym. Sci., Part A: Polym. Chem. 2015, 53, 2462.

(10) (a) Parker, W. L.; Johnson, F. J. Org. Chem. 1973, 38, 2489. (b) Walraven, H. G. M.; Pandit, U. K. Tetrahedron 1980, 36, 321. (c) Strunz, G. M.; Lal, G. S. Can. J. Chem. 1982, 60, 2528.

(11) (a) Weise, C. F.; Lauridsen, V. H.; Rambo, R. S.; Iversen, E. H.; Olsen, M. L.; Jørgensen, K. A. J. Org. Chem. 2014, 79, 3537. (b) Jiang, H.; Albrecht, Ł.; Jørgensen, K. A. Chem. Sci. 2013, 4, 2287. (c) Albrecht, Ł.; Dickmeiss, G.; Acosta, F. C.; RodríguezEscrich, C.; Davis, R. L.; Jørgensen, K. A. J. Am. Chem. Soc. 2012, $134,2543$.

(12) (a) Malerich, J. P.; Hagihara, K.; Rawal, V. H. J. Am. Chem. Soc. 2008, 130, 14416. (b) For a review on squaramides, see: Alemán, J.; Parra, A.; Jiang, H.; Jørgensen, K. A. Chem. Eur. J. 2011, 17, 6890. (c) Mechanism: Kótai, B.; Kardos, G.; Hamza, A.; Farkas, V.; Pápai, I.; Soós, T. Chem. Eur. J. 2014, 20, 5631.

(13) (a) Li, B.-J.; Jiang, L.; Liu, M.; Chen, Y.-Ch.; Ding, L.-S.; Wu, Y. Synlett 2005, 603. (b) Vakulya, B.; Varga, S.; Csámpai, A.; Soós, T. Org. Lett. 2005, 7, 1967. (c) McCooey, S. H.; Connon, S. J. Angew. Chem. Int. Ed. 2005, 44, 6367. (d) Ye, J.; Dixon, D. J.; Peter, S.; Hynes, P. S. Chem. Commun. 2005, 4481. (e) Mechanism: Hamza, A.; Schubert, G.; Soós, T.; Pápai, I. J. Am. Chem. Soc. 2006, $128,13151$.
(14) Berkes, B.; Ozsváth, K.; Molnár, L.; Gáti, T.; Holczbauer, T.; Kardos, G.; Soós, T. Chem. Eur. J. 2016, 22, 18101.

(15) For details see the Supporting Information.

(16) (a) Dedeoglu, B.; Catak, S.; Houk, K. N.; Aviyente, V. ChemCatChem 2010, 2, 1122. (b) Blise, K.; Cvitkovic, M. W.; Gibbs, N. J.; Roberts, S. F.; Whitaker, R. M.; Hofmeister, G. E.; Kohen, D. J. Org. Chem. 2017, 82, 1347.

(17) Mechanism of lactone hydrolysis: Gómez-Bombarelli, R.; Calle, E.; Casado, J. J. Org. Chem. 2013, 78, 6868.

(18) Frisch, M. J.; Trucks, G. W.; Schlegel, H. B.; Scuseria, G. E.; Robb, M. A.; Cheeseman, J. R.; Scalmani, G.; Barone, V.; Petersson, G. A.; Nakatsuji, H.; Li, X.; Caricato, M.; Marenich, A. V.; Bloino, J.; Janesko, B. G.; Gomperts, R.; Mennucci, B.; Hratchian, H. P.; Ortiz, J. V.; Izmaylov, A. F.; Sonnenberg, J. L.; Williams-Young, D.; Ding, F.; Lipparini, F.; Egidi, F.; Goings, J.; Peng, B.; Petrone, A.; Henderson, T.; Ranasinghe, D.; Zakrzewski, V. G.; Gao, J.; Rega, N.; Zheng, G.; Liang, W.; Hada, M.; Ehara, M.; Toyota, K.; Fukuda, R.; Hasegawa, J.; Ishida, M.; Nakajima, T.; Honda, Y.; Kitao, O.; Nakai, H.; Vreven, T.; Throssell, K.; Montgomery, J. A. Jr.; Peralta, J. E.; Ogliaro, F.; Bearpark, M. J.; Heyd, J. J.; Brothers, E. N.; Kudin, K. N.; Staroverov, V. N.; Keith, T. A.; Kobayashi, R.; Normand, J.; Raghavachari, K.; Rendell, A. P.; Burant, J. C.; Iyengar, S. S.; Tomasi, J.; Cossi, M.; Millam, J. M.; Klene, M.; Adamo, C.; Cammi, R.; Ochterski, J. W.; Martin, R. L.; Morokuma, K.; Farkas, O.; Foresman, J. B.; Fox, D. J. Gaussian 16, Revision B.01; Gaussian, Inc: Wallingford CT, 2016. 